\title{
A driven two-dimensional granular gas with Coulomb friction
}

\author{
Olaf Herbst ${ }^{\text {a) }}$ \\ Institut für Theoretische Physik, Friedrich-Hund-Platz 1, 37077 Göttingen, Germany \\ Raffaele Cafiero \\ P.M.M.H., Ecole Supèrieure de Physique et de Chimie Industrielles (ESPCI), \\ 10, rue Vauquelin-75251 Paris cedex 05, France \\ Annette Zippelius \\ Institut für Theoretische Physik, Friedrich-Hund-Platz 1, 37077 Göttingen, Germany \\ Hans Jürgen Herrmann \\ P.M.M.H., Ecole Supèrieure de Physique et de Chimie Industrielles (ESPCI), \\ 10, rue Vauquelin-75251 Paris cedex 05, France \\ and Institute for Computer Physics, Pfaffenwaldring 27, 70569 Stuttgart, Germany \\ Stefan Luding ${ }^{\text {b) }}$ \\ Institute for Computer Physics, Pfaffenwaldring 27, 70569 Stuttgart, Germany \\ and Particle Technology, DelftChemTech, TU Delft, Julianalaan 136, 2628 BL Delft, The Netherlands
}

(Received 10 January 2005; accepted 28 June 2005; published online 3 October 2005)

\begin{abstract}
We study a homogeneously driven granular gas of inelastic hard particles with rough surfaces subject to Coulomb friction. The stationary state as well as the full dynamic evolution of the translational and rotational granular temperatures are investigated as a function of the three parameters of the friction model. Four levels of approximation to the (velocity-dependent) tangential restitution are introduced and used to calculate translational and rotational temperatures in a mean field theory. When comparing these theoretical results to numerical simulations of a randomly driven monolayer of particles subject to Coulomb friction, we find that already the simplest model leads to qualitative agreement, but only the full Coulomb friction model is able to reproduce/predict the simulation results quantitatively for all magnitudes of friction. In addition, the theory predicts two relaxation times for the decay to the stationary state. One of them corresponds to the equilibration between the translational and rotational degrees of freedom. The other one, which is slower in most cases, is the inverse of the common relaxation rate of translational and rotational temperatures. (C) 2005 American Institute of Physics. [DOI: 10.1063/1.2049277]
\end{abstract}

\section{INTRODUCTION}

Granular media are collections of macroscopic particles with arbitrary shape, rough surfaces, and dissipative interactions. ${ }^{1-4}$ Many phenomenona are well reproduced by model granular media, where spheres are used instead of other, possibly more realistic shapes. In order to study such model systems, kinetic theories ${ }^{4-16}$ and numerical simulations ${ }^{4,15,17-23}$ have been applied for special boundary conditions and a variety of interesting experiments have been performed, see, for example, Refs. 24-28. The dynamics of the system is usually assumed to be dominated by instantaneous two-particle collisions. These collisions are dissipative and frictional, and conserve linear and angular momentum while energy is not conserved. In the simplest model, one describes inelastic collisions by a normal restitution coefficient $r$ only. However, surface roughness and friction are important, ${ }^{10,13,20-22,29}$ since they allow for an exchange of translational and rotational energy and influence the overall dissipation. In the standard approach, ${ }^{5,10,22}$ surface roughness is accounted for by a constant tangential restitution coeffi-

\footnotetext{
${ }^{a)}$ Electronic-mail: olaf.herbst@gmx.net

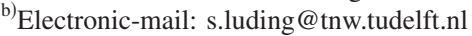

cient $r_{t}$, which is defined in analogy to $r$ in the tangential direction. A more realistic friction law involves the Coulomb friction coefficient $\mu,{ }^{17,30-32}$ so that the tangential restitution $r_{t}(\gamma)$ depends on the impact angle $\gamma$, i.e., the angle between the contact normal and the relative velocity of the contact points.

Recently, Jenkins and Zhang ${ }^{14}$ proposed a kinetic theory for frictional, nearly elastic spheres in the limit of small friction coefficient $\mu$. They introduced an effective coefficient of normal restitution by approximately relating the rotational temperature to the translational one. Thereby the kinetic theory for slightly frictional, nearly elastic spheres has the same structure as that for frictionless spheres. Also for small $\mu$, Goldhirsch et al. ${ }^{16}$ showed that an infinite number of spindependent number densities is needed to describe the dynamics of frictional spheres and that the distribution of rotational velocities is non-Gaussian. A mean field theory for threedimensional cooling systems of rough particles with Coulomb friction was proposed in Ref. 13 and found to be in very good agreement with computer simulations for a wide range of parameters. A systematic theoretical study of driven systems over the whole range of dissipation and friction parameters is not available to our knowledge. 
In the following, we propose a mean field (MF) theory of homogeneously driven rough particles that accounts for Coulomb friction (i.e., a nonconstant $r_{t}$ ) on different levels of refinement. The most accurate description parallels the threedimensional (3D) results ${ }^{13}$ for freely cooling systems. In addition, we present different levels of approximation to the full model and discuss their shortcomings in MF theory. The homogeneous driving used here is the same as in other recent studies of driven systems. ${ }^{15,29}$

To test our analytical results we have performed numerical simulations of a randomly driven monolayer of spheres, using an Event Driven (ED) algorithm ${ }^{21,22,29,33}$. One key result is that, via $r_{t}(\gamma)$, all parameters of the collision model affect the evolution of the translational and rotational degrees of freedom (temperatures) of the system. Only the full MF theory is able to quantitatively predict the system behavior for the whole parameter range.

This paper is organized as follows. The model system is introduced in Sec. II. The distribution of impact angles, as affected by translational and rotational degrees of freedom, is computed in Sec. III. The standard approach with constant tangential restitution is briefly reviewed, before we introduce three levels of approximation and the full MF theory in Sec. IV. In Sec. V we discuss the stationary state and in Sec. VI the dynamic evolution towards the stationary state. In both sections we compare the predictions of full MF theory and its approximations to simulations. Finally, we present a summary and conclusions in Sec. VII.

\section{MODEL}

The model system contains $N$ three-dimensional spheres of diameter $2 a$, mass $m$, and moment of inertia $I$ interacting via a hard-core potential. The particles are confined to a twodimensional (2D) square with periodic boundary conditions. The linear box size is $L$ and the area (volume) $V=L^{2}$. The moment of inertia can be expressed using the shape factor

$$
q:=\frac{I}{m a^{2}}
$$

For spheres with a homogeneous mass distribution $q=2 / 5$. Inelasticity and roughness are described by a coefficient of normal restitution $r$, the Coulomb friction law with coefficient of friction $\mu$, and a coefficient of tangential restitution $r_{t}$ which depends on $r, \mu$, and the impact angle $\gamma$ for sliding contacts, or on a maximum tangential restitution $r_{t}^{m}$ for sticking contacts, when some "tangential elasticity" becomes important. In a collision of two particles $i=1$ and 2 with positions $\boldsymbol{r}_{i}$, contact normal $\boldsymbol{n}=\left(\boldsymbol{r}_{1}-\boldsymbol{r}_{2}\right) /(2 a)$, angular velocities $\boldsymbol{\omega}_{i}$ and relative translational velocity $\boldsymbol{v}_{12}=\boldsymbol{v}_{1}-\boldsymbol{v}_{2}$ (see Fig. 1), their velocities after the collision are related to the velocities before the collision, through a collision matrix ${ }^{20,30,32,34}$ which is derived from the conservation laws for linear and angular momentum, energy/dissipation balance, and Coulomb's friction law. This three parameter model is able to reproduce the experimental measurements on colliding spheres of various materials. $^{30,35}$

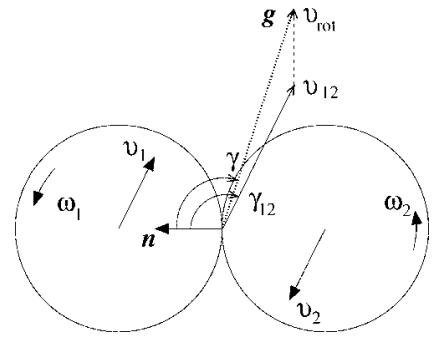

FIG. 1. Schematic drawing of two-particle contact in the center of mass reference frame. Shown are the relative velocity $g$ of the contact points, the impact angle $\gamma$ of the contact points, and the angle $\gamma_{12}$ between the relative translational velocity of the particles and their contact normal.

\section{A. Collision rules}

The collision rules are most transparent when written in terms of the relative velocity of the contact point in the center-of-mass reference frame

$$
\boldsymbol{g}=\boldsymbol{v}_{1}-\boldsymbol{v}_{2}-a\left(\boldsymbol{\omega}_{1}+\boldsymbol{\omega}_{2}\right) \times \boldsymbol{n} .
$$

We decompose $\boldsymbol{g}=\boldsymbol{g}_{n}+\boldsymbol{g}_{t}$ into its normal and tangential components with respect to $\boldsymbol{n}, \boldsymbol{g}_{n}=(\mathbf{g} \cdot \mathbf{n}) \mathbf{n}$ and $\mathbf{g}_{t}=\mathbf{g}-\mathbf{g}_{n}$. The change of normal momentum of particle 1 , denoted by $\Delta \boldsymbol{P}^{(n)}$ is the same as for smooth particles

$\Delta \boldsymbol{P}^{(n)}=-(m / 2)(1+r) \mathbf{g}_{n}$.

The change of tangential momentum

$$
\Delta \boldsymbol{P}^{(t)}=-\frac{q}{q+1} m\left(1+r_{t}\right) \boldsymbol{g}_{t}
$$

is, in general, a function of the impact angle $\gamma$. Coulomb friction can be expressed ${ }^{34}$ in terms of a coefficient of tangential restitution

$$
r_{t}(\gamma)=\min \left[r_{t}^{C}(\gamma), r_{t}^{m}\right]
$$

which is a function of the impact angle $\gamma$ between $\boldsymbol{g}$ and $\boldsymbol{n}$. Here $r_{t}^{m}$ is the coefficient of maximum tangential restitution, with $-1 \leqslant r_{t}^{m} \leqslant 1$ to ensure that energy is not created. The quantity $r_{t}^{C}(\gamma)$ is determined using Coulomb's law

$$
r_{t}^{C}(\gamma)=-1-\frac{q+1}{q} \mu(1+r) \cot \gamma,
$$

with the impact angle $\pi / 2<\gamma \leqslant \pi$ so that $\cos \gamma=\boldsymbol{g} \cdot \boldsymbol{n} /|\boldsymbol{g}|$ is always negative. ${ }^{20,30,32}$ Here, we have simplified the tangential contacts in the sense that exclusively either Coulomb friction applies, i.e., $\Delta P^{(t)}=\mu \Delta P^{(n)}$, or constant tangential restitution with the maximum tangential restitution coefficient $r_{t}^{m}$. Coulomb friction is effective when the relative tangential velocity is large, whereas tangential restitution applies for low tangential velocities.

Note that in the general case $\boldsymbol{v}_{\text {rot }}=-a\left(\boldsymbol{\omega}_{1}+\boldsymbol{\omega}_{2}\right) \times \boldsymbol{n} \neq 0$, so that the angle $\gamma_{12}$ between the contact normal $\boldsymbol{n}$ and the relative translational velocity $\boldsymbol{v}_{12}=\boldsymbol{v}_{1}-\boldsymbol{v}_{2}$ is different from the impact angle $\gamma$ of the contact points, see Figs. 1 and 2. In the following we will refer to $\gamma$ when we talk about the impact angle. 


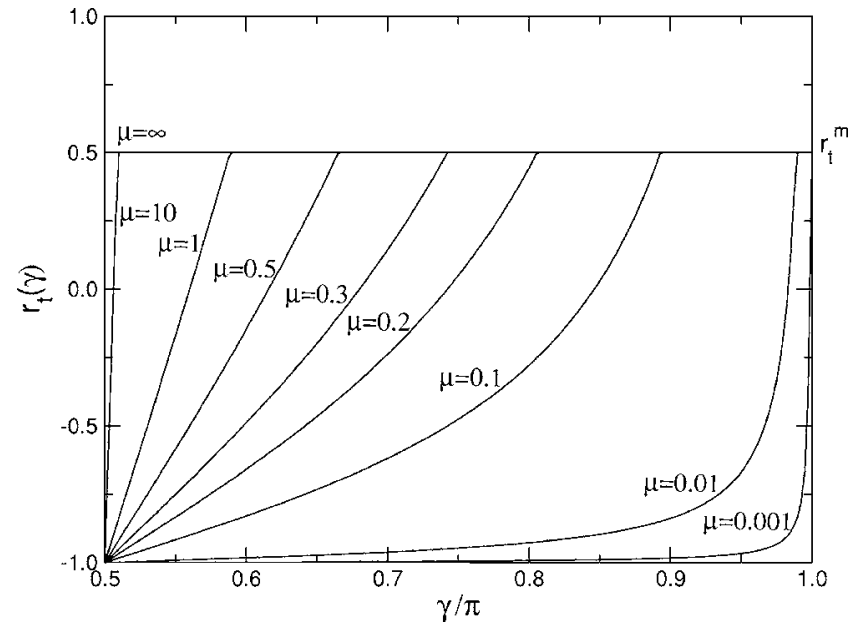

FIG. 2. Tangential restitution $r_{t}$ as function of the impact angle $\gamma$ for different values of the coefficient of friction $\mu$.

\section{B. Driving model}

The driving of a granular material can be realized by moving walls, see Ref. 1 and references therein, corresponding to a local heating, ${ }^{36-39}$ or the system can alternatively be driven by a global homogeneous, random energy source in different variations. ${ }^{11,12,29,40-42}$ We choose homogeneous translational driving here and modify the velocity of particle $i$ at each time of agitation $t$ such that

$$
\boldsymbol{v}_{i}^{\prime}(t)=\boldsymbol{v}_{i}(t)+v_{\mathrm{dr}} \boldsymbol{\xi}_{i}(t),
$$

where the prime on the left-hand side indicates the value after the driving event. Measuring masses in units of the particle mass $m$, the driving velocity $v_{\mathrm{dr}}$ sets the time (velocity) scale and defines the driving temperature $T_{\mathrm{dr}}:=m v_{\mathrm{dr}}^{2}$. The components of the vector $\boldsymbol{\xi}_{i}(t), \xi_{i, x}(t)$ and $\xi_{i, y}(t)$, are uncorrelated Gaussian random numbers with zero mean and variance

$$
\left\langle\xi_{i, k}(t) \xi_{j, l}\left(t^{\prime}\right)\right\rangle_{\{\xi\}}=\delta_{i j} \delta_{k l} \delta\left(t-t^{\prime}\right),
$$

where $\delta_{i j}$ and $\delta_{k l}$ are Kronecker deltas and $\delta\left(t-t^{\prime}\right)$ is the Dirac delta function. The stochastic driving rule in Eq. (7) leads to an average rate of change of temperature

$$
\Delta T / \Delta t=H_{\mathrm{dr}}, \quad \text { with } H_{\mathrm{dr}}=f_{\mathrm{dr}} T_{\mathrm{dr}},
$$

after every driving time step $\Delta t=f_{\mathrm{dr}}^{-1}$.

\section{Simulations}

We have performed simulations of a randomly driven monolayer of spheres, using an Event Driven (ED) algorithm, ${ }^{20,21,29,43}$ and compared the results with the MF predictions, see also Refs. 11, 13, 29, and 42. Every simulation is equilibrated without driving with $r=1$ and in the smooth surface limit $r_{t}^{m}=-1$. Then inelasticity, friction, and driving are switched on, according to the rules defined above. The problem of the inelastic collapse characteristic of the ED algorithm, ${ }^{44,45}$ is handled by using normal restitution coefficients dependent on the time elapsed since the last event. ${ }^{46-48}$ The frequency of driving is chosen such that it is larger than or comparable to the typical collision frequency per particle, both initially and in steady state. Varying the driving frequency to much larger values did not affect the simulation results, whereas the use of a much smaller driving rate caused different results due to the slow input of energy.

\section{IMPACT-ANGLE PROBABILITY DISTRIBUTION}

In the following we shall discuss various levels of approximation to the collision rules given in Eqs. (5) and (6). One possibility to simplify the collision rules is to consider tangential restitution averaged over all impact angles $\gamma$, thereby reducing the problem to one with a constant coefficient of tangential restitution. For that purpose we need to know the probability distribution of impact angles.

The assumption of "molecular chaos" implies a homogeneous distribution of the collision parameter $b$ $=2 a \sin \gamma_{12}$ which is simply related to the angle $\gamma_{12}$ between the relative translational velocity $\boldsymbol{v}_{12}$ and the contact normal $\boldsymbol{n}$ according to $\cos \gamma_{12}=\boldsymbol{v}_{12} \cdot \boldsymbol{n} /\left|\boldsymbol{v}_{12}\right|$, see Fig. 1. Hence the probability distribution of $\sin \gamma_{12}$ is constant, $P^{\prime}{ }_{12}\left(\sin \gamma_{12}\right)$ $\equiv 1$. (The prime indicates probability functions of the sine or the cosine of the angle.) A uniform probability $P^{\prime}$ implies for the distribution of the angle $P_{12}\left(\gamma_{12}\right)=-\cos \gamma_{12}$, so that grazing contacts appear less probable than central collisions when a fixed interval $\mathrm{d} \gamma_{12}$ is considered. The uniform $P^{\prime}{ }_{12}\left(\sin \gamma_{12}\right)$ is in agreement with our numerical data, see Fig. 3.

In general, the impact angle $\gamma$ between the relative velocity of the contact point $\boldsymbol{g}$ and the contact normal $\boldsymbol{n}$ is different from the angle $\gamma_{12}$ between the relative translational velocity $\boldsymbol{v}_{12}$ and the contact normal $\boldsymbol{n}$, as displayed in Fig. 1. The two angles are identical only in the case of smooth particles or in the limit of vanishingly small rotational velocities. In the general case we compute $P^{\prime}(\cos \gamma)$ by averaging over all binary collisions

$$
P^{\prime}(\cos \gamma)=\left\langle\delta\left(\cos \gamma-\frac{\boldsymbol{g} \cdot \boldsymbol{n}}{|\boldsymbol{g}|}\right)\right\rangle_{\text {coll }} .
$$

Analytically this average can only be computed approximately. We assume that the translational and rotational velocities of the colliding particles are distributed according to Gaussians with a temperature $T_{\text {tr }}$ for the translational and a temperature $T_{\text {rot }}$ for the rotational velocities. Within this approximation the above average is given explicitly by

$$
P^{\prime}(\cos \gamma)=\frac{J\left(\delta\left(\cos \gamma-\frac{\boldsymbol{g} \cdot \boldsymbol{n}}{|\boldsymbol{g}|}\right)\right)}{J(1)}
$$

with the phase space integral

$$
J(X)=\int d \Gamma_{1} d \Gamma_{2}\left(\boldsymbol{v}_{12} \cdot \boldsymbol{n}\right) \Theta\left(-\boldsymbol{v}_{12} \cdot \boldsymbol{n}\right) \delta\left(\left|\boldsymbol{r}_{12}\right|-2 a\right) X,
$$

where $X=X\left(\Gamma_{1}, \Gamma_{2}\right)$, and the phase space element

$$
d \Gamma_{k}=d^{2} r_{k} d^{2} v_{k} d \omega_{k} e^{-m v_{k}^{2} /\left(2 T_{\text {tr }}\right)} e^{-I w_{k}^{2} /\left(2 T_{\text {rot }}\right)}
$$

for $k=1,2$. 

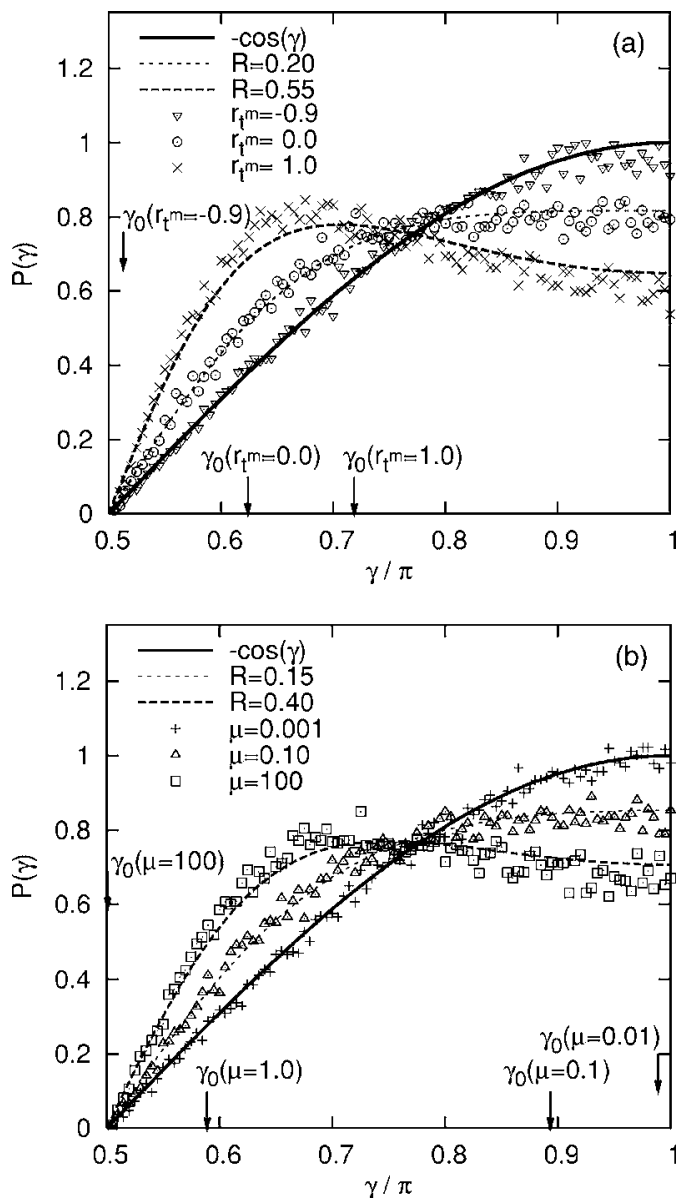

FIG. 3. Plots of the probability distribution of $\gamma$ from simulations (symbols) and from Eq. (12) with $R$ values from the simulations. The arrows indicate the corresponding $\gamma_{0}$, while the parameters are (a) $r=0.95, \mu=0.5$ and variable $r_{t}^{m}$, and (b) $r=0.95, r_{t}^{m}=0.4$ and variable $\mu$.

The remaining integrals can be computed analytically, yielding the following expression for the impact angle distribution:

$$
P(\gamma)=-\frac{(1+R / q) \cos \gamma}{\left(1+[R / q] \cos ^{2} \gamma\right)^{3 / 2}} .
$$

Here we have introduced the ratio of rotational and translational temperatures $R:=T_{\text {rot }} / T_{\text {tr }}$ and recall $q=I /\left(m a^{2}\right)$. The probability distribution $P(\gamma)$ is compared to the results of our simulations in Fig. 3; reasonably good agreement is observed. With increasing rotational velocities, contacts with large $g_{t}$ (small $\gamma$ ) become more and more frequent due to the increasing rotational contribution. On the other hand, collisions with vanishing $g_{t}$ (large $\gamma$ ) become less probable, since the rotational contribution leads to a net increase of $g_{t}$.

\section{DIFFERENTIAL EQUATIONS IN MEAN FIELD THEORY APPROXIMATIONS}

In the following we present different approximations for frictional particles, referred to as models A-E. Model A is the well known model using constant coefficients of normal and tangential restitution cf., e.g., Refs. 5 and 10. Model E implements Coulomb friction as introduced by Walton. ${ }^{17}$ While model A is the mean field solution for rough particles with a constant coefficient of tangential restitution, model $\mathrm{E}$ is the mean field solution for particles with Coulomb friction. Models B through D are approximations to model E that may be simpler to deal with but have significant shortcomings.

The starting point of our mean field approach is the theory of Ref. 10 for a freely cooling gas of rough particles with a constant coefficient of tangential restitution $\left(r_{t}\right.$ $=$ const, corresponding to the limit $\mu \rightarrow \infty$ ). The theory is based on a pseudo-Liouville-operator formalism and on the assumption of (i) a homogeneous state, (ii) independent Gaussian probability distributions of all degrees of freedom, i.e., all components of the translational and the rotational velocities, and (iii) the assumption of "molecular chaos," i.e., subsequent collisions are uncorrelated. The agreement with simulations is very good as long as the above assumptions are valid. ${ }^{21}$

The main outcome of this approach is a set of coupled time evolution equations for the translational and rotational MF temperatures $T_{\text {tr }}$ and $T_{\text {rot }}$ (for definitions cf. Eq. (A6) in the Appendix) which can be extended to also describe arbitrary energy input (driving). ${ }^{15,29,42}$ Given the random driving temperature $T_{\mathrm{dr}}$ and an energy input rate $f_{\mathrm{dr}}$, as defined above, one just has to add the positive rate of change of translational energy $H_{\mathrm{dr}}$, see Eq. (9), to the system of equations. ${ }^{29}$

\section{A. Model A: Constant tangential restitution $r_{t}=r_{t}^{m}$}

We recall the results of the mean field theory for the model with a constant coefficient of tangential restitution which is obtained from the general case in the limit $\mu \rightarrow \infty$ [see Eq. (14) in Ref. 21]. The system of coupled equations reads in $2 \mathrm{D}$ :

$$
\begin{aligned}
& \frac{d}{d t} T_{\mathrm{tr}}(t)=H_{\mathrm{dr}}+G\left[-A T_{\mathrm{tr}}^{3 / 2}+B T_{\mathrm{tr}}^{1 / 2} T_{\mathrm{rot}}\right], \\
& \frac{d}{d t} T_{\mathrm{rot}}(t)=2 G\left[B^{\prime} T_{\mathrm{tr}}^{3 / 2}-C T_{\mathrm{tr}}^{1 / 2} T_{\mathrm{rot}}\right] .
\end{aligned}
$$

Note the choice of signs which lead to positive coefficients. Based on more physical arguments, $A$ quantifies the dissipation of translational energy, $B$ and $B^{\prime}$ correspond to the interchange of energy between the translational and rotational degrees of freedom, and $C$ describes the dissipation of rotational energy. The coefficient $G$ sets the time scale of the system, i.e., the collision rate (per particle) $\tau^{-1}=(1 / 2) G T_{\mathrm{tr}}^{1 / 2}$, with

$$
G=\frac{8}{a \sqrt{\pi m}} \nu g_{2 a}(\nu) .
$$

Here $g_{2 a}(\nu)$ denotes the pair correlation function at contact. In the approximation proposed by Henderson, ${ }^{5,49-52} g_{2 a}(\nu)$ $=(1-7 \nu / 16) /(1-\nu)^{2}$, it depends only on the $2 \mathrm{D}$ volume fraction of the granular gas $\nu=\pi a^{2} N / V$. The four constants $A, B, B^{\prime}$, and $C$ read in this limit

$$
A=A_{r}+A_{\eta_{0}}, \quad A_{r}:=\frac{1-r^{2}}{4},
$$




$$
\begin{aligned}
& A_{\eta_{0}}:=\frac{\eta_{0}}{2}\left(1-\eta_{0}\right), \\
& B^{\prime}=B=B_{\eta_{0}}:=\frac{\eta_{0}^{2}}{2 q},
\end{aligned}
$$

and

$$
C=C_{\eta_{0}}:=\frac{\eta_{0}}{2 q}\left(1-\frac{\eta_{0}}{q}\right) .
$$

It is useful to define a function

$$
\eta\left(r_{t}\right):=\frac{q\left(1+r_{t}\right)}{2(q+1)}, \quad \text { for } 0 \leqslant \eta\left(r_{t}\right) \leqslant \frac{q}{q+1}<1,
$$

which has to be evaluated at constant tangential restitution $r_{t}=r_{t}^{m}$ in the limit $\mu \rightarrow \infty$ :

$$
\eta_{0}:=\eta\left(r_{t}^{m}\right)=\frac{q\left(1+r_{t}^{m}\right)}{2(q+1)} .
$$

\section{B. Model B: Simplified mean tangential restitution $r_{t}=\left\langle r_{t}\right\rangle_{12}$}

A first step beyond the above theory with a constant $\eta_{0}=\eta\left(r_{t}^{m}\right)$, is the replacement of $r_{t}(\gamma)$ by its average

$$
\left\langle r_{t}\right\rangle=\int_{\pi / 2}^{\pi} d \gamma P(\gamma) r_{t}(\gamma)
$$

The integral over $\gamma$ from $\pi / 2$ to $\pi$, has to be split into two parts, one corresponding to the range $\pi / 2<\gamma<\gamma_{0}$ for which there is Coulomb sliding with $r_{t}$ given by Eq. (6), and a second part corresponding to the range $\gamma_{0} \leqslant \gamma \leqslant \pi$, for which there is sticking with constant $r_{t}=r_{t}^{m}$ (see Fig. 2). The critical angle $\gamma_{0}$ is given by

$$
c:=-\cot \gamma_{0}=\frac{q\left(1+r_{t}^{m}\right)}{\mu(q+1)(1+r)}>0 .
$$

To simplify the computation, we use the approximation $P(\gamma) \approx P_{12}(\gamma)=-\cos (\gamma)$, such that

$$
\left\langle r_{t}\right\rangle_{12}=-1+\frac{q+1}{q}(1+r) \mu \ln (c+f)
$$

with the abbreviation

$$
f:=\sqrt{1+c^{2}} .
$$

The averaged coefficient of tangential restitution $\left\langle r_{t}\right\rangle_{12}$ must be inserted into $\eta$ in Eq. (19). Thus we obtain the same set of coefficients as in Eqs. (15)-(18) with $\eta_{0}$ replaced by

$$
\eta_{1}:=\eta\left(\left\langle r_{t}\right\rangle_{12}\right)=\frac{\eta_{0}}{c} \ln (c+f) .
$$

In this approach, only the average value of $r_{t}$ is considered and fluctuations of $r_{t}$ with $\gamma$ are neglected. Furthermore the difference between $\gamma$ and $\gamma_{12}$ has been ignored in the averaging procedure. In contrast to model A this is the simplest model to incorporate the coefficient of Coulomb friction $\mu,\left\langle r_{t}\right\rangle_{12}=\left\langle r_{t}\right\rangle_{12}(\mu)$.

\section{Model C: Mean tangential restitution $r_{t}=\left\langle r_{t}\right\rangle(R)$}

In model $\mathrm{C}$ we again replace $r_{t}(\gamma)$ by its average but use the correct impact angle probability distribution function $P(\gamma)$ from Eq. (12) in the averaging procedure. The result is an $R$-dependent averaged coefficient of tangential restitution

$$
\begin{aligned}
\left\langle r_{t}\right\rangle(R)= & -1+\frac{q+1}{q} \frac{1+r}{4} \frac{\mu}{x} \\
& \times \ln \left\{\frac{\frac{R}{q}(f-c)^{2}\left(x \tilde{f}-f+c \frac{R}{q}\right)}{\left(x \tilde{f}-f-c \frac{R}{q}\right)^{2}\left(x \tilde{f}+f-c \frac{R}{q}\right)}\right\}
\end{aligned}
$$

with

$$
\begin{aligned}
& x^{2} \equiv x^{2}(R):=1+R / q, \\
& \tilde{f} \equiv \tilde{f}(R):=\sqrt{1+x^{2} c^{2}},
\end{aligned}
$$

and $f$ defined in Eq. (24). Note that $x$ is an implicit function of time through $R$. For $R \rightarrow 0(x \rightarrow 1)$ Eq. (26) reduces to Eq. (23) - as expected. For $R \rightarrow \infty(x \rightarrow \infty)$ there is no friction and $\left\langle r_{t}\right\rangle(R) \rightarrow-1$.

We formally get the same differential equations (13) but with nonconstant coefficients $A=A(R), B^{\prime}=B=B(R)$, and $C$ $=C(R)$ which are obtained by replacing $\eta_{0}$ by $\eta\left(\left\langle r_{t}\right\rangle(R)\right)$ in Eqs. (15)-(18). These coefficients are implicitly time dependent via $R$.

\section{Constant tangential restitution limit}

In the limit $\mu \rightarrow \infty, c \rightarrow 0$. In that case model $\mathrm{C}$ reduces to model A.

\section{Weak friction limit}

For $\mu \rightarrow 0, c \rightarrow \infty$ we recover smooth spheres with $\left\langle r_{t}\right\rangle$ $\rightarrow-1$. A series expansion to lowest order in $\mu$ (equivalent to lowest order in $c^{-1}$ ) of Eq. (26) reads

$$
\begin{aligned}
\left\langle r_{t}\right\rangle(R)= & -1+\frac{q+1}{q}(1+r) \frac{\mu}{x}\{|\ln (\mu)|+\ln (x) \\
& \left.+\ln \left(\frac{2 \eta_{0}}{1+r}\right)\right\}+\mathcal{O}\left(\mu^{3}\right),
\end{aligned}
$$

expressed in terms of $x$ and $\mu$.

As long as $x$ stays finite (which is the case for a driven system) the leading order is thus $\mu|\ln (\mu)|$ for small $\mu$. For $x \rightarrow 1$, Eq. (29) yields the same result as Eq. (23) in leading order in $c^{-1}$.

\section{Comparison of model $B$ and model $C$}

Due to the implicit nature of model $\mathrm{C}$ it is rather difficult to work out its predictions, e.g., for the ratio of temperatures. Therefore, we present here the mean tangential restitution from models A, B, and C in Fig. 4. Note that $\left\langle r_{t}\right\rangle$ for model $\mathrm{C}$ depends not only explicitly on $\mu$ but also implicitly through $R$. To keep the discussion simple, we present results only for some constant, representative values of $R$. The mean restitution for large $R$ is smaller (or equivalently, the corresponding $\mu$ is larger) than for small $R$. Models $\mathrm{B}$ and $\mathrm{C}$ become indistinguishable in the limit $R \rightarrow 0$, as expected. 


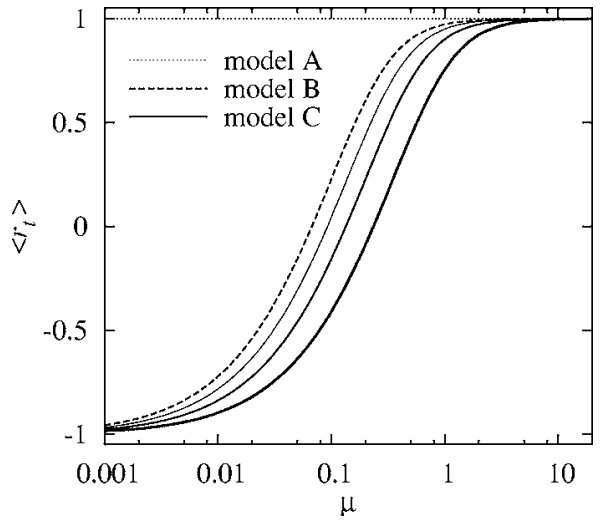

FIG. 4. Expected mean tangential restitution, $\left\langle r_{t}\right\rangle$, as function of the friction coefficient $\mu$ for models A, B, and C. The parameters used are $r=0.95, r_{t}^{m}$ $=1.0$ (for A, B) and different $R=1.0,0.40$, and 0.15 (model C: solid lines from right to left).

\section{Model D: Variable (simplified) tangential restitution $r_{t}\left(\gamma_{12}\right)$}

In this section and in the following one, we discuss a coefficient of tangential restitution which depends on $\gamma$. Model $\mathrm{D}$ is defined by approximating $\gamma \approx \gamma_{12}$, which is strictly true only for $R \rightarrow 0$ or $\mu \rightarrow 0$ (or equivalently $\left.r_{t}^{m} \rightarrow-1\right)$. We again obtain the same differential equations (13) for $T_{\text {rot }}$ and $T_{\text {tr }}$ with the coefficients

$$
\begin{aligned}
& A=A_{\mu}=A_{r}+\left[A_{\eta_{0}}+A^{*}\right] / f^{3}, \\
& B=B_{\mu}=\left[B_{\eta_{0}}+B^{*}\right] / f, \\
& B^{\prime}=B^{\prime}{ }_{\mu}=\left[B_{\eta_{0}}+B^{\prime *}\right] / f^{3}, \\
& C=C_{\mu}=\left[C_{\eta_{0}}+C^{*}\right] / f,
\end{aligned}
$$

and $f$ defined in Eq. (24). The terms that originate from Coulomb sliding are denoted by an asterisk and are given explicitly by

$$
\begin{aligned}
& B^{*}=\frac{\eta_{0}^{2} c^{2}}{2 q(f+1)^{2}}, \\
& B^{\prime *}=(2 f+1) B^{*}, \\
& A^{*}=\eta_{0} c^{2} / 2-q B^{*}, \\
& C^{*}=\left(\eta_{1} f-\eta_{0}-2 B^{*}\right) /(2 q),
\end{aligned}
$$

expressed in terms of $f$ [cf. Eq. (24)], $\eta_{0}$ [cf. Eq. (20)], $\eta_{1}$ [cf. Eq. (25)], and $q$ [cf. Eq. (1)]. The terms $B^{*}$ and $B^{\prime *}$ are strictly positive, while the dissipation correction terms $A^{*}$ and $C^{*}$, in principle, can change sign. Note also that $B^{*}$ and
$B^{\prime *}$ are not identical here. All coefficients depend on the system parameters only. They are constants in time-in contrast to model $\mathrm{C}$ (and $\mathrm{E}$ as will be shown later).

\section{Constant tangential restitution limit}

In the limit $\mu \rightarrow \infty$, one has $c \rightarrow 0$, i.e., $f \rightarrow 1$, and all correction terms $\left\{A^{*}, B^{*}, B^{\prime *}, C^{*}\right\} \rightarrow 0$ so that one obtains Eqs. (13)-(18). Note in particular that the coefficients $B_{\mu}$ and $B^{\prime}{ }_{\mu}$ are equal only in the limit $\mu \rightarrow \infty .{ }^{10}$

\section{Weak friction limit}

In the limit $\mu \rightarrow 0(c \rightarrow \infty, f \rightarrow c)$, the lowest order expansion in $c^{-1}$ leads to an approximation of the coefficients in Eqs. (30), where we have used $\eta_{0} / c=\mu(1+r) / 2$ :

$$
\begin{aligned}
& B_{\mu}=\frac{\eta_{0}}{q} \frac{1+r}{2} \mu+\mathcal{O}\left(\mu^{2}\right), \\
& B^{\prime}{ }_{\mu}=\frac{1}{q}\left(\frac{1+r}{2}\right)^{2} \mu^{2}+\mathcal{O}\left(\mu^{3}\right), \\
& A_{\mu}=A_{r}+\frac{1+r}{4} \mu+\mathcal{O}\left(\mu^{2}\right), \\
& C_{\mu}=\frac{1}{2 q} \frac{1+r}{2} \mu\left[|\ln (\mu)|+\ln \left(\frac{4 \eta_{0}}{1+r}\right)-2 \frac{\eta_{0}}{q}\right]+\mathcal{O}\left(\mu^{2}\right) .
\end{aligned}
$$

From Eqs. (32), we learn that $B^{\prime}{ }_{\mu}$ is second order in $\mu$, whereas $B_{\mu}$ is first order in $\mu$, reflecting an asymmetry in the energy transfer rates. On the other hand, $A_{\mu} \approx A_{r}$ is almost constant, whereas $C_{\mu}$ depends on $\mu$ logarithmically which is an artifact of our approximation $\gamma_{12} \sim \gamma$, see Eq. (35) below.

\section{E. Model E: Variable (exact) tangential restitution $r_{t}(\gamma)$}

The final step of refinement of the MF theory is to use $r_{t}(\gamma)$, instead of $r_{t}\left(\gamma_{12}\right)$, to compute the coefficients. This is the full mean field theory. The calculation is similar to the one for 3D in Ref. 13 and is presented in the Appendix. We obtain the following coefficients, to be inserted into Eqs. (13):

$$
\begin{aligned}
& A=\widetilde{A}_{\mu}(R)=A_{r}+\left[A_{\eta_{0}}+\widetilde{A}^{*}\right] / \widetilde{f}^{3}, \\
& B=\widetilde{B}_{\mu}(R)=\left[B_{\eta_{0}}+\widetilde{B}^{*}\right] / \widetilde{f}^{3}, \\
& B^{\prime}=\widetilde{B}_{\mu}^{\prime}(R)=\left[B_{\eta_{0}}+\widetilde{B}^{\prime *}\right] / \widetilde{f}^{3}, \\
& C=\widetilde{C}_{\mu}(R)=\left[C_{\eta_{0}}+\widetilde{C}^{*}\right] / \widetilde{f}^{3},
\end{aligned}
$$

with $\tilde{f}, x$, and $c$ defined in Eqs. (28), (27), and (22), respectively. The new correction terms are in detail: 


$$
\begin{aligned}
& \widetilde{B}^{*}=-\eta_{0} c^{2} /(2 q), \\
& \widetilde{B}^{\prime *}=\frac{(2 \tilde{f}+1)\left(\eta_{0} c x^{2}\right)^{2}}{2 q(\tilde{f}+1)^{2}}, \\
& \widetilde{A}^{*}=-q\left(\widetilde{B}^{*}+\widetilde{B}^{\prime *}\right), \\
& \widetilde{C}^{*}=-x^{2} \widetilde{B}^{*},
\end{aligned}
$$

with $q$ and $x$ as introduced in Eqs. (1) and (27). Interestingly, we find now a negative $\widetilde{B}^{*}$ together with positive coefficients $\widetilde{B}^{\prime *}$ and $\widetilde{C}^{*}$; only $\widetilde{A}^{*}$ can be both positive and negative. Like in model $\mathrm{C}$ but in contrast to models $\mathrm{A}, \mathrm{B}$, and $\mathrm{D}$, here the coefficients are implicit functions of time, again.

In conclusion, models D and E appear similar in shape but there are several striking differences: (i) The division by $f$ and $f^{3}$ in model $\mathrm{D}$ is in contrast with the division by $\widetilde{f}^{3}$ in model $\mathrm{E}$, (ii) the term $B^{*}$ in model $\mathrm{D}$ is always positive, while $\widetilde{B}^{*}$ in model $\mathrm{E}$ is always negative, (iii) the sign of $C^{*}$ in model $\mathrm{D}$ is not determined a priori, while the term $\widetilde{C}^{*}$ is always positive, (iv) among the correction terms of model E, only $\widetilde{B}^{*}$ is independent of $R$, and (v) the more refined theory appears in a simpler form, especially the term $\widetilde{C}^{*}$.

\section{Constant tangential restitution limit}

The limit of constant tangential restitution can be reached by taking the limit $\mu \rightarrow \infty$. In this case $c \rightarrow 0, \tilde{f} \rightarrow 1$ and thus all additional coefficients $\widetilde{A}^{*}, \widetilde{B}^{*}, \widetilde{B}^{\prime *}$, and $\widetilde{C}^{*}$ vanish such that Eqs. (13)-(18) are recovered.

\section{Weak friction limit}

In the limit $\mu \rightarrow 0 \quad(c \rightarrow \infty, \tilde{f} \rightarrow x c)$ an expansion to the lowest order in $\mu$ leads to an approximation of the coefficients in Eqs. (33) when we remember that $\eta_{0} / c=(1$ $+r) \mu / 2$ :

$$
\begin{aligned}
& \widetilde{B}_{\mu}(R)=-\frac{1}{2 q x^{3}} \frac{1+r}{2} \mu+\mathcal{O}\left(\mu^{3}\right), \\
& \widetilde{B}_{\mu}^{\prime}(R)=\frac{1}{q}\left(\frac{1+r}{2}\right)^{2} \mu^{2}+\mathcal{O}\left(\mu^{3}\right), \\
& \widetilde{A}_{\mu}(R)=A_{r}-q\left(\widetilde{B}_{\mu}(R)+\widetilde{B}^{\prime}{ }_{\mu}(R)\right)+\mathcal{O}\left(\mu^{3}\right), \\
& \widetilde{C}_{\mu}(R)=-x^{2} \widetilde{B}_{\mu}(R)+\mathcal{O}\left(\mu^{3}\right) .
\end{aligned}
$$

Since $x=x(R)$ approaches 1 in the weak friction limit, both $\widetilde{B}_{\mu}(R)$ and $\widetilde{C}_{\mu}(R)$ are proportional to $\mu$ in leading order. To lowest order in $\mu$, Eq. (35) predicts $\widetilde{A}_{\mu}(R)=A_{r}+\mathcal{O}(\mu)$, i.e., proportional to $\mu^{0}$, while $\widetilde{B}^{\prime}{ }_{\mu}(R)$ is proportional to $\mu^{2}$.

For $\mu \ll 1$, Eqs. (13) with (35) simplify to

$$
\frac{d}{d t} T_{\mathrm{tr}}(t)=H_{\mathrm{dr}}-G T_{\mathrm{tr}}^{3 / 2}\left(\frac{1-r^{2}}{4}+\mathcal{O}(\mu)\right),
$$

which means that in the limit of low friction the differential equations for $T_{\text {tr }}$ and $T_{\text {rot }}$ decouple. In the nondriven case this leads to surviving rotational energy (not shown), similar to Refs. 13 and 16.

\section{STEADY STATE}

Before discussing the approach to the stationary state in the next chapter, we first elucidate the stationary state and compare results of our simulations to various levels of refinement of the mean field theory.

\section{A. Analytical results}

By imposing $(d / d t) T_{\text {tr }}^{\text {stat }}=0$ and $(d / d t) T_{\text {rot }}^{\text {stat }}=0$ one gets the steady state values of the rotational and the translational temperatures. For models A, B and D, the coefficients in the differential equation do not depend on $R$ (or $x$ ). Therefore the solution is simply

$$
T_{\text {rot }}^{\text {stat }}=R^{\text {stat }} T_{\text {tr }}^{\text {stat }}, \quad \text { and } \quad T_{\text {tr }}^{\text {stat }}=\left(\frac{H_{\mathrm{dr}}}{G \mathcal{I}}\right)^{2 / 3},
$$

with

$$
R^{\text {stat }}=B^{\prime} / C, \quad \text { and } \quad \mathcal{I}=A-B R^{\text {stat }},
$$

as discussed in more detail for all models in the following.

\section{Model A}

For model A, the steady state ratio of rotational to translational energies is

$$
R^{\text {stat }}=\frac{q \eta_{0}}{q-\eta_{0}}
$$

and the energy dissipation factor is

$$
\mathcal{I}=\frac{1-r^{2}}{4}-\frac{\eta_{0}}{2}\left(1-\eta_{0}\right)-\frac{\eta_{0}^{3}}{2\left(q-\eta_{0}\right)} \text {. }
$$

Note here again that model A does not contain any dependence on the coefficient of friction $\mu$.

\section{Model B}

Model B evolves from model A, by just replacing $\eta_{0}$ by $\eta_{1}(\mu)=\left(\eta_{0} / c\right) \ln (c+f)$ from Eq. (25) in the above Eqs. (39) and (40), so that, e.g.,

$$
R^{\mathrm{stat}}=\frac{q \eta_{1}}{q-\eta_{1}}=\frac{q\left(\eta_{0} / c\right) \ln (c+f)}{q-\left(\eta_{0} / c\right) \ln (c+f)} .
$$

In the limit of small $\mu \ll 1$, the leading order terms are $R^{\text {stat }} \approx(1+r) \mu|\ln \mu| / 2$ and $\mathcal{I} \approx\left(1-r^{2}\right) / 4+\mathcal{O}(\mu|\ln \mu|)$.

\section{Model D}

From model D, the following, more complex terms are obtained: 


$$
R_{\mu}^{\mathrm{stat}}=\frac{B_{\mu}^{\prime}}{C_{\mu}}=\frac{\left[B_{\eta_{0}}+B^{\prime \prime}\right]}{\left[C_{\eta_{0}}+C^{*}\right]} \frac{1}{f^{2}} \underset{\mu \ll 1}{\approx}(1+r) \mu|\ln \mu|
$$

and

$$
\mathcal{I}_{\mu}=A_{\mu}-B_{\mu} R_{\mu}^{\mathrm{stat}} \underset{\mu \ll 1}{\approx} \frac{1-r^{2}}{4}+\mathcal{O}(\mu),
$$

so that, asymptotically for $\mu \ll 1$, model D leads to behavior similar to that of model B.

\section{Model E}

Formally, we can write down Eqs. (37) for model E, too. Instead of using Eqs. (38), $R^{\text {stat }}$ must be extracted (numerically) from Eq. (A22) where the left-hand side vanishes in the stationary case. It can be shown analytically that there is always a unique solution - in contrast to the freely cooling case. ${ }^{13}$ With the solution for $R^{\text {stat }}$ at hand, Eq. (A19) (with a vanishing left-hand side) can be written in the form $T_{\text {tr }}^{\text {stat }}$ $=\left(H_{\mathrm{dr}} / G \mathcal{I}\right)^{2 / 3}$ again where $\mathcal{I}$ is a nonlinear function of $R^{\text {stat }}$ whose particular form can be easily seen from Eq. (A19), cf. Appendix.

\section{Models $C$ and $E$ for small $\mu$}

For models $\mathrm{C}$ and $\mathrm{E}$ the coefficients in the differential equations $d o$ depend on $R$, so that the steady state values have to be computed numerically for a general choice of parameters. Analytical results can only be achieved in the limit $\mu \ll 1$, where we can use the expansions of the coefficients introduced in Sec. IV C and IV E.

For model $\mathrm{C}$ we obtain to lowest order in $\mu$, the dissipation factor $\mathcal{I} \approx A_{r}$ and, using $\eta_{2}=\left(\eta_{0} / 2\right) \ln c$,

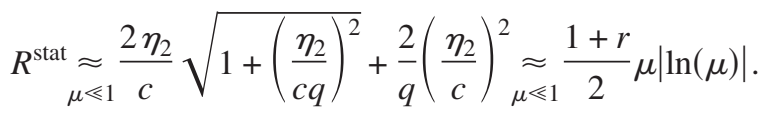

For model $\mathrm{E}$ we find again $\mathcal{I} \approx A_{r}$ and

$$
R_{\mu \ll 1}^{\text {stat }} \underset{\mu}{\approx} \frac{2 \eta_{0}}{c} \sqrt{1+\left(\frac{\eta_{0}}{c q}\right)^{2}}+\frac{2}{q}\left(\frac{\eta_{0}}{c}\right)^{2} \underset{\mu \ll 1}{\approx}(1+r) \mu,
$$

very similar in shape to the result from model $\mathrm{C}$, besides the $\operatorname{logarithm} \ln c$ that is hidden in the definition of $\eta_{2}$. This leads to the qualitative difference in asymptotic behavior between models $\mathrm{C}$ and $\mathrm{E}$ : The correct asymptotic behavior for small $\mu$ is $R^{\text {stat }} \propto \mu$. Note again that the more refined model $\mathrm{E}$ leads to a simpler analytical result than the approximated model C.

\section{Discussion}

The expansions for small $\mu \ll 1$ show that the result for $R^{\text {stat }}$ based on model E, see Eq. (42), disagrees with all other models. In model $\mathrm{E}$ we find that $R^{\text {stat }}$ vanishes linearly as $\mu \rightarrow 0$, whereas models A-D predict a slower decrease, encoded in the $\mu|\ln \mu|$ dependence. Models A and B have the same analytical form for $R^{\text {stat }}$ if expressed in terms of $\eta_{0}$ for model A and in terms of $\eta_{1}$ for model B. Similarly, models C and $\mathrm{E}$ have the same functional dependence on $\eta$, if $\eta_{2}$ is used for model $\mathrm{C}$ and $\eta_{0}$ for model $\mathrm{E}$. The comparison of the models for arbitrary values of $\mu$ will be given in the next subsection, where we also present the results of our simulations and compare them to the predictions of the various mean field models.

\section{B. Comparison with simulations}

In this subsection, the steady state predictions from our models are confronted with the numerical simulation results. Note that we present results for rather high densities and dissipation, where our assumptions about homogeneity of the system and the Gaussian shape of the velocity distributions is not strictly true anymore. However, we want to stress the point that the present theory is astonishingly close to the numerical simulation with experimentally relevant parameters even when the most basic assumptions are somewhat questionable.

\section{Variation of $r_{t}^{m}$}

In Figs. 5(a)-5(c), the stationary rotational and translational temperatures and their ratio $R$ are compared for $r=0.95, \mu=0.5$ and different values of $r_{t}^{m}$; note that the data in (a) and (b) are scaled with the expression for $\mu=0$. The symbols correspond to simulation data, with the error bars showing the standard deviation from the mean values. The lines correspond to different refinements of the theoretical approaches, i.e., models A, B, D, and E.

For $r_{t}^{m} \approx-1$, the simulations agree with all theoretical predictions; for $r_{t}^{m} \approx 1$, large discrepancies are evident. The more refined a model used, the better the quality of agreement. The qualitative behavior of the data is best captured by model $\mathrm{E}$, and we relate the remaining quantitative deviations to the fact that the simulations involve rather high density $\nu$ and comparatively strong dissipation $r$.

\section{Variation of $\mu$-translational temperature}

In Fig. 6 we plot the translational temperature in the same way as in Fig. 5(a), but now, we keep the values $r_{t}^{m}=0.4$ (a) and $r_{t}^{m}=1.0$ (b) fixed and vary $\mu$. Furthermore, we compare data for $r=0.99$ and $r=0.95$ in one plot and observe satisfactory agreement between simulation results and the full mean field theory, model E. (The predictions from models A and B are only shown for $r=0.99$.)

For (realistic) values of $r_{t}^{m}=0.4$, see Fig. 6(a), one obtains a transition from the $\mu=0$ limit to the $\mu \rightarrow \infty$ value of the kinetic energy, over three orders of magnitude in $\mu$, whereas for $r_{t}^{m}=1.0$, see Fig. 6(b), the kinetic energy first decays with $\mu$ but then increases again to the stationary state temperature of smooth particles, since no energy is dissipated due to tangential friction for $\mu \rightarrow \infty$ and $r_{t}^{m}=1.0$.

We remark that model A, with $r_{t}=r_{t}^{m}$ and the limit $\mu$ $\rightarrow \infty$ is inadequate to model the $\mu$ dependency of the data; it only gives the $\mu \rightarrow \infty$ limit, as expected. Approach B only shows qualitative agreement with our simulation data, whereas theory D shows good quantitative agreement for small $\mu$. The agreement seems better for weak normal dissipation $r=0.99$, as compared to the cases with $r=0.95$. The 

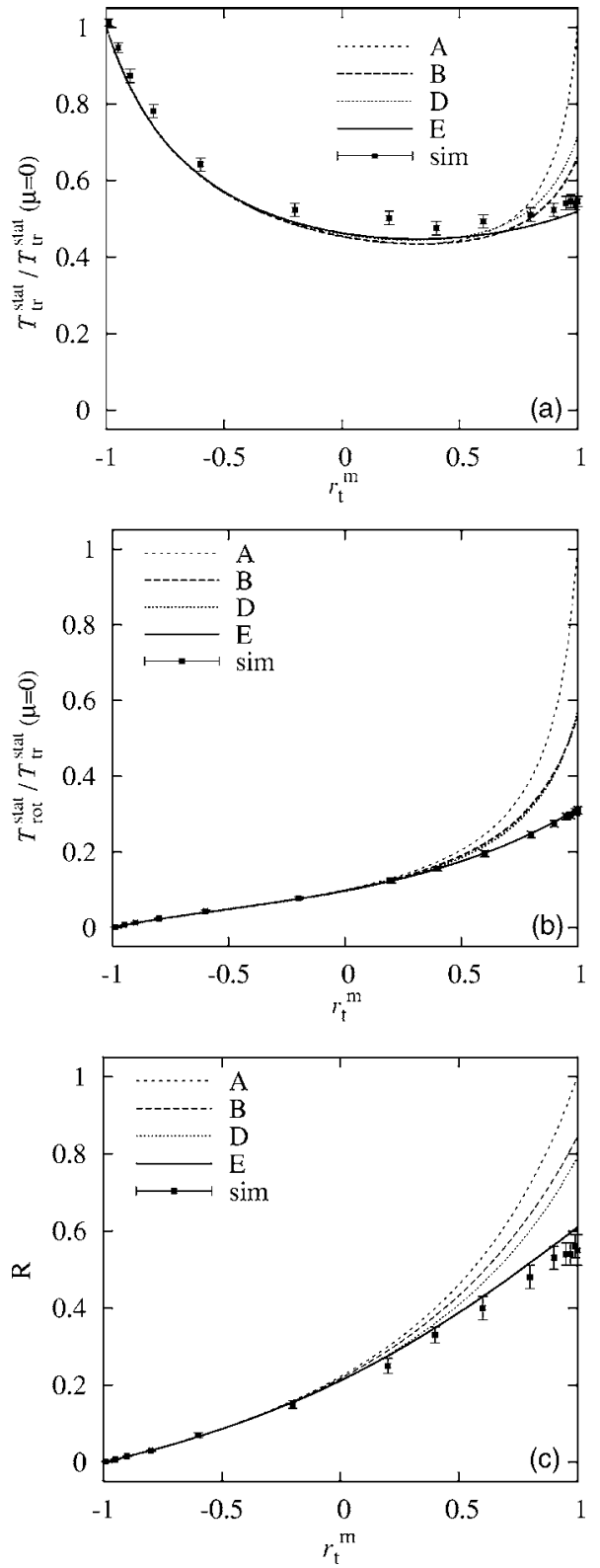

FIG. 5. Simulation results (symbols) and theory (lines) for the parameters $\nu=0.34, N=11025, r=0.95$, and $\mu=0.5$, plotted against the maximum tangential restitution $r_{t}^{m}$. (a) Translational temperature $T_{\text {tr }}^{\text {stat }}$, and (b) rotational temperature $T_{\text {rot }}^{\text {stat }}$, plotted against $r_{t}^{m}$, and scaled by $T_{\text {tr }}^{\text {stat }}(\mu=0)$, the mean field value for smooth particles. (c) Ratio of rotational and translational temperature $R$, plotted against $r_{t}^{m}$.

deviations between simulations and model D in the intermediate range of $\mu$ are due to values of $R$ of the order of unity, for which the assumption $\gamma_{12} \approx \gamma$ is not true, as pointed out above.

For weaker normal dissipation $r$, one obtains a stronger reduction of the translational temperature in the range of strongest total dissipation (around $\mu \approx 0.4$ ). This is due to the comparatively stronger contribution of tangential dissipation. However, as in the previous subsection, the agreement between simulations and model $\mathrm{E}$ is satisfactory, especially for $r \rightarrow 1$.
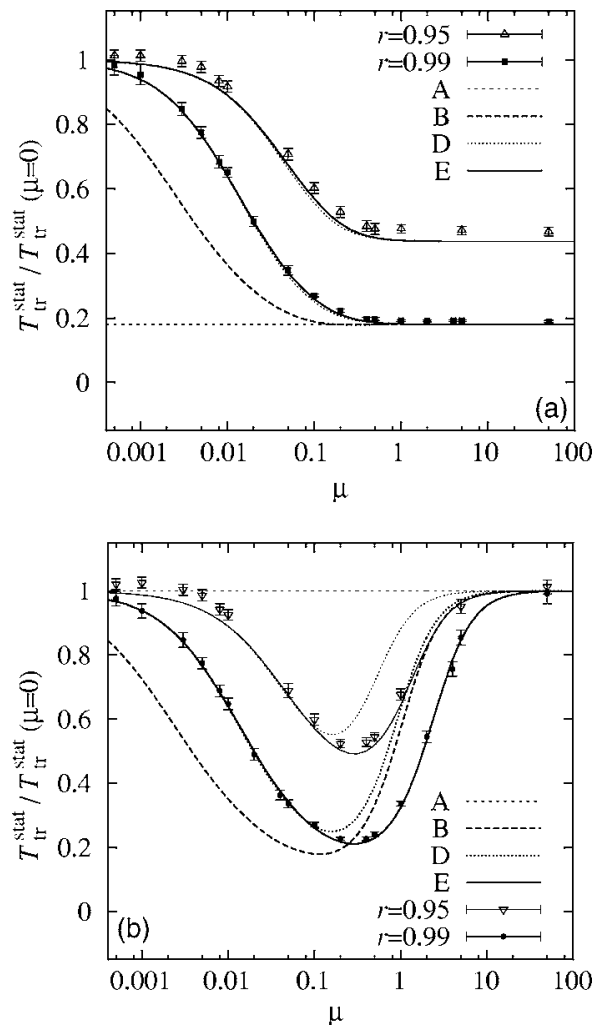

FIG. 6. Translational temperature $T_{\text {tr }}^{\text {stat }}$ scaled by the mean field value for smooth particles $T_{\mathrm{tr}}^{\mathrm{stat}}(\mu=0)$, plotted against $\mu$, for the parameters as in Fig. 5. The tangential restitution coefficients are fixed to (a) $r_{t}^{m}=0.4$, and (b) $r_{t}^{m}=1$.0. Data with normal restitution $r=0.99$ (solid symbols and thick lines) and $r=0.95$ (open symbols and thin lines) are compared. Models A and B are shown for $r=0.99$ only.

\section{Variation of $\mu$-rotational temperature}

In Fig. 7 we plot the ratio of rotational and translational temperatures in the same way as in Fig. 5(c), but now, like in Fig. 6, we keep the values $r_{t}^{m}=0.4$ (a) and $r_{t}^{m}=1.0$ (b) fixed and vary $\mu$. Also here, we compare data for $r=0.99$ and $r=0.95$ in one plot. For the values of $r_{t}^{m}$ examined (see Fig. 7) one observes a smooth transition of $R$ over about three orders of magnitude in $\mu$, from the value $R=0$ (in the limit $\mu=0$ ) to the value $R=r_{t}^{m}$ (in the limit $\mu \rightarrow \infty$ ). Note that the observation $R=r_{t}^{m}$ is coincidence, since the correct asymptotic result for large $\mu$ is $R=2\left(1+r_{t}^{m}\right) /\left(9-5 r_{t}^{m}\right)$. Again, the agreement between simulations and model $\mathrm{E}$ is impressive.

All models agree qualitatively in the large $\mu$ limit, even though the quantitative agreement with simulations is again best caught by model E, as can be seen in Fig. 8 .

The remaining question is the asymptotic behavior for very small $\mu$, as can be viewed in Fig. 9, and as discussed theoretically in Sec. V A. The quantitative behavior of $R$ for small $\mu$ is tested by a power law fit of the numerical values, according to an expression $R=b \mu^{\alpha}$. The fit yields $\alpha=1.00(4)$ for $r=0.99$ and $r_{t}^{m}=0.4$ or $r_{t}^{m}=1.0$, and $\alpha=0.99$ (4) for $r=0.95$ and $r_{t}^{m}=0.4$ or $r_{t}^{m}=1.0$. Thus the asymptotic behavior is proportional to $\mu$, in excellent qualitative and quantitative agreement with the prediction of model E. 

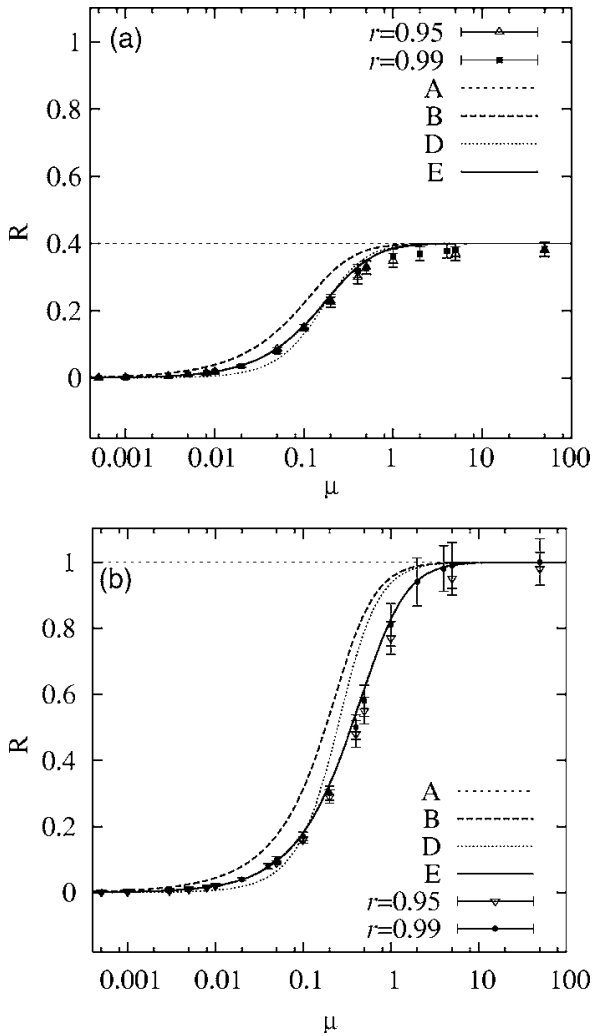

FIG. 7. $R$ plotted against $\mu$, for the same parameters as in Fig. 6 . The tangential restitution coefficients are again fixed to (a) $r_{t}^{m}=0.4$, and (b) $r_{t}^{m}$ $=1.0$.

\section{APPROACH TO STEADY STATE}

\section{A. Close to steady state}

Provided the system is sufficiently close to steady state, we can linearize the set of Eqs. (13) around $T_{\mathrm{tr}}^{\text {stat }}$ and $T_{\text {rot }}^{\text {stat }}$. This is particularly simple for models A, B, and D, where the coefficients in the differential equation do not depend on $R$ and hence can be solved analytically for the stationary state. We set $T_{\text {tr }}(t)=T_{\text {tr }}^{\text {stat }}\left[1+\delta T_{\text {tr }}(t)\right]$ and $T_{\text {rot }}(t)=T_{\text {rot }}^{\text {stat }}\left[1+\delta T_{\text {rot }}(t)\right]$ and obtain the linearized dynamic equations

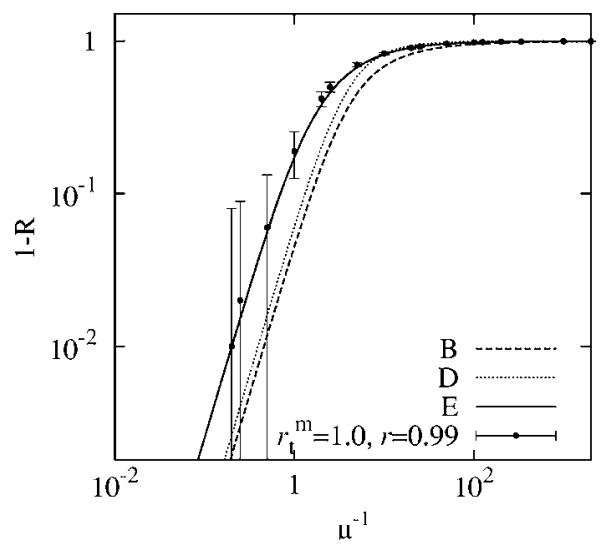

FIG. 8. Deviation from equipartition, $1-R$, plotted against the inverse friction coefficient, $\mu^{-1}$, for simulations from Fig. 6(b). Note the doublelogarithmic scale of this plot.

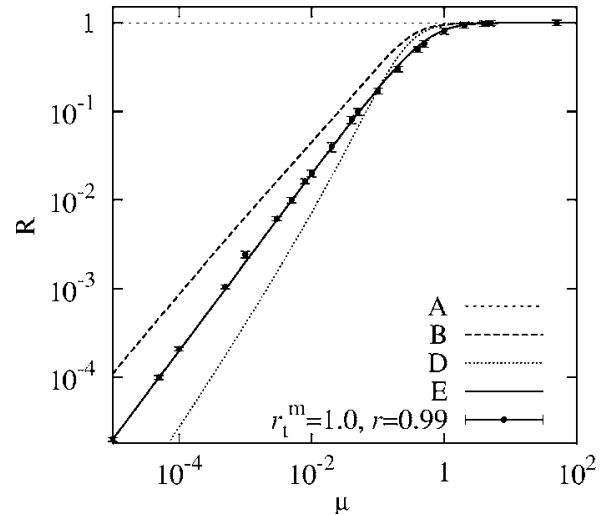

FIG. 9. Ratio of rotational and translational temperature, $R$, plotted against $\mu$, for some simulations from Fig. 6(b). Note the double-logarithmic scale of this plot.

$$
\begin{aligned}
& \frac{d}{d t} \delta T_{\text {tr }}=G T_{\text {tr }}^{\text {stat }}\left\{\left(\frac{3}{2} A+\frac{B B^{\prime}}{2 C}\right) \delta T_{\text {tr }}+\frac{B B^{\prime}}{C} \delta T_{\text {rot }}\right\}, \\
& \frac{d}{d t} \delta T_{\text {rot }}=2 G C T_{\text {tr }}^{\text {stat }}\left\{\delta T_{\text {tr }}-\delta T_{\text {rot }}\right\} .
\end{aligned}
$$

This set of linear equations is easily solved to yield two relaxation rates $\lambda_{1}$ and $\lambda_{2}$. In a stable stationary state they must be positive and they are. We present here only results for the simplest model (A) and postpone the general discussion to the next paragraph, where the full dynamic evolution toward steady state will be examined.

In Fig. 10, we plot the two relaxation rates as a function of $r_{t}^{m}$ for a fixed value of $r=0.95$. In the limit of smooth spheres one of the rates vanishes because the rotational energy is conserved in that limit. For $r_{t}^{m} \sim-0.84$ the two rates are equal and for increasing $r_{t}^{m}$ the difference between the two rates increases monotonically with $r_{t}^{m}$, such that for perfectly rough spheres the larger rate is about 14 times the smaller one. Such a pronounced separation of time scales is familiar from the cooling dynamics of the same model, see Ref. 13. There it was shown that the ratio of translational to rotational energy, $R$, relaxes fast to its stationary value, whereas both the translational as well as the rotational energy decay on the same, much longer time scale. This point

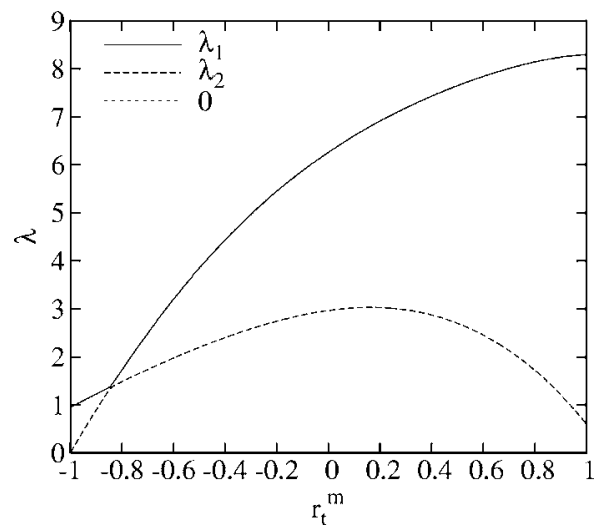

FIG. 10. Relaxation rates $\lambda_{1,2}$, close to steady state for $r=0.95$ as a function of $r_{t}^{m}$. 

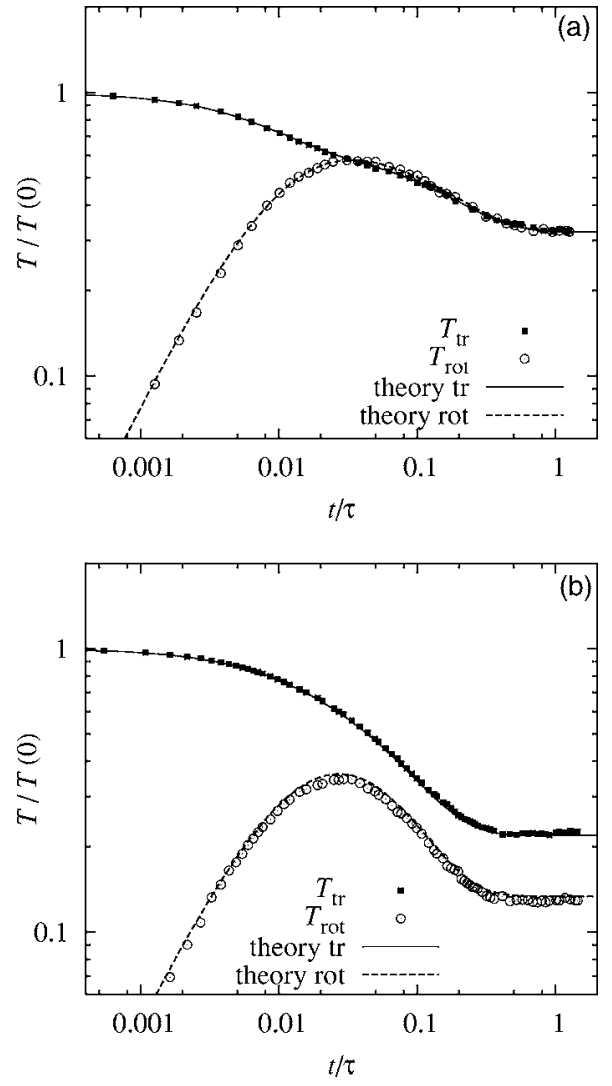

FIG. 11. Evolution of temperatures with rescaled time, with $\tau^{-1}$ $=(1 / 2) G T_{\mathrm{tr}}(0)^{1 / 2}$, for simulations with $N=11025, \nu=0.0866, r=0.95, r_{t}^{m}$ $=1.0$, and (a) $\mu=\infty$, (b) $\mu=0.5$. Note that there are no fit parameters.

will be discussed in a more general setting (model $\mathrm{E}$ and relaxation from an arbitrary initial condition) in the subsequent paragraph.

\section{B. Full dynamic evolution}

In Fig. 11, the full dynamic evolution of the translational and rotational temperatures with time is shown for two simulations with $N=11025, \nu=0.0866, r=0.95, r_{t}^{m}=1.0$, and different values for the coefficient of friction. In both situations, the agreement between simulations and the numerical solution for the full MF theory, model E, is good-not only concerning the limiting values and the asymptotes, but also the time dependence during the two regimes (i) equilibration between $T_{\text {tr }}$ and $T_{\text {rot }}$, and (ii) approach to final steady state.

\section{SUMMARY AND DISCUSSION}

In summary, a dynamic MF theory for the full time evolution of the translational and rotational temperatures of a homogeneously driven two-dimensional granular gas has been presented. Particle collisions were modeled using the Walton model, ${ }^{17}$ i.e., with normal dissipation, tangential restitution (sticking) and Coulomb friction (sliding). The Walton model can be formulated in terms of a coefficient of tangential restitution, which depends on the impact angle $\gamma$. Using a pseudo-Liouville operator we have computed the distribution of impact angles as well as the mean field dynamics and steady state values of the translational and rotational temperatures.

In addition to the complete mean field theory of the Walton model ("model E"), we discussed three levels of approximation in order to simplify the differential equations of the time evolution. The crudest approximations including Coulomb friction ("model B" and "model C") assume that an effective constant tangential restitution exists and can be computed by averaging over the angular distribution of impact angles. For model $\mathrm{C}$ this averaged coefficient depends on the current values of the translational and rotational temperatures and thus on time. Even simpler is model B where the rotational contribution to the impact angle is neglected, leading to a coefficient of tangential restitution that only depends on global system parameters. The closest approximation ("model D") to the full mean field theory ("model E") keeps the dependence of $r_{t}(\gamma)$ on the impact angle $\gamma$ but, like for model $\mathrm{B}$, the contribution of the rotation of the particles to the impact angle is neglected.

The predictions of the increasingly refined models of frictional dissipation as well as the full MF theory have been compared to simulations of a randomly driven monolayer of spheres using an Event Driven algorithm. Emphasis has been put on the stationary state which is characterized by two temperatures, $T_{\mathrm{tr}}$ and $T_{\text {rot }}$, one for the translational and one for the rotational degrees of freedom. Guided by the MF approach we discovered a rich phenomenology like a nontrivial dependence of the stationary state temperatures on the model parameters. For example, the translational temperature is nonmonotonic as a function of maximal tangential restitution $r_{t}^{m}$ and also nonmonotonic as a function of Coulomb friction $\mu$, provided $r_{t}^{m}$ is sufficiently large.

All models predict steady state values of the translational and rotational temperatures, which are considerably improved as compared to the model without friction ("model A"), which assumes constant tangential restitution (see Figs. 6 and 7). All approximations A-E agree in the limit of large friction, where the tangential restitution becomes independent of the impact angle (see Fig. 2). Qualitative agreement between models B-D and simulations is achieved also for intermediate values of $\mu$. However, in the limit $\mu \rightarrow 0$ all approximations break down and only the complete mean field solution ("model E") is in agreement with the simulations (see Fig. 9). In particular model E predicts the linear dependence of the ratio of temperatures, $R=T_{\text {rot }} / T_{\text {tr }}$, on the friction coefficient $\mu$ that is observed in the simulations and was used in Ref. 14 to derive an approximate kinetic theory of frictional particles.

Sticking contacts become more important relative to sliding contacts for fixed $\mu$ and decreasing $r_{t}^{m}$. In this regime models B and D seem reasonable, but lead to poor quantitative agreement as $r_{t}^{m}$ approaches 1 . The full mean field theory ("model E") leads to reasonable agreement for all values of $r_{t}^{m}$. For weak dissipation, $r \rightarrow 1$, the agreement is very good-for stronger dissipation, we relate the deviations to the failure of both the homogeneity assumption and the molecular chaos assumption made.

Linearizing the dynamic MF equations around the steady 
state leads to an eigenvalue problem with two relaxation rates, one of them being related to the equilibration between the translational and the rotational degrees of freedom, while the other one controls the approach of the system to its steady state. For strong coupling, the former process is much faster, so that there is a clear separation of time scales, which has been discussed already for a freely cooling system in the absence of driving.

In conclusion, realistic Coulomb friction turned out to be a subtle problem as only the full mean field theory of the Walton model predicts the effects of friction for all values of $\mu$ and $r_{t}^{m}$. All simplifications are both qualitatively and quantitatively wrong in some parameter range. So far our investigations have been based on the Walton model which has been shown to be in good agreement with experiments for two-particle collisions. ${ }^{30}$ It would be interesting to test the robustness of our results to modifications of this model. This is beyond our present paper but could, in principle, be addressed within the presented formalism. Furthermore, our studies can easily be extended to three-dimensional systems or more complex ones, like, e.g., a polydisperse mixture of frictional particles with different material properties. Other driving mechanisms could be employed as well, in particular driving through the boundaries (e.g., vibrating walls or sheared walls) or simple shear. We expect our results to apply for those systems, too, as long as the driving is sufficiently strong in order to keep the system approximately homogeneous. After this work was completed we learned about a related work by Yoon and Jenkins ${ }^{53}$ that is similar in spirit.

\section{ACKNOWLEDGMENTS}

This work has been supported by the European network project FMRXCT980183 (RC) and the DFG (Deutsche Forschungsgemeinschaft) through SFB 602 (O.H. and A. Z), Grant No. Zi209/6-1. (A.Z.) S.L. was also supported by the DFG, Grant No. Lu450/9-1, and by FOM (Stichting Fundamenteel Onderzoek der Materie, The Netherlands) as financially supported by NWO (Nederlandse Organisatie voor Wetenschappelijk Onderzoek).

\section{APPENDIX: DERIVATION OF THE DIFFERENTIAL EQUATIONS FOR MODEL E}

The details of the derivation of the coefficients in Eqs. (34) and (33) for model E in Sec. IV E will be shown. The calculations are performed using a pseudo-Liouville operator formalism. ${ }^{13,21,54}$ They are very similar to the ones in three dimensions. ${ }^{13}$ First, we briefly recall the pseudo-Liouville operator formalism.

Let the vectors of position, translational and rotational velocity of a particle $k$, in a two-dimensional plane $(x, y)$ with only vertical spin $(z)$, be defined as $\boldsymbol{r}_{k}=\left(r_{k, x}, r_{k, y}, 0\right)$, $\boldsymbol{v}_{k}=\left(v_{k, x}, v_{k, y}, 0\right)$, and $\boldsymbol{\omega}_{k}=\left(0,0, \omega_{k}\right)$.

The time evolution of a dynamic variable $A(t)$ that depends on time only through the positions and velocities of $N$ particles, can be determined by means of a pseudo-Liouville operator $\mathcal{L}_{+}$for $t>0$ :

$$
A(t)=\exp \left(i \mathcal{L}_{+} t\right) A(0)
$$

The pseudo-Liouville operator $\mathcal{L}_{+}$consists of three parts $\mathcal{L}_{+}=\mathcal{L}_{0}+\mathcal{L}^{\prime}{ }_{+}+\mathcal{L}_{+}^{H}$. The last part, $\mathcal{L}_{+}^{H}$, describes the homogeneous driving, the first one, $\mathcal{L}_{0}$, describes the free streaming of particles

$$
\mathcal{L}_{0}=-i \sum_{k=1}^{N} \boldsymbol{v}_{k} \cdot \nabla_{\boldsymbol{r}_{k}},
$$

and the second one, $\mathcal{L}^{\prime}{ }_{+}=\frac{1}{2} \Sigma_{l \neq k} T_{+}^{k l}$ describes hard-core collisions of two particles

$$
T_{+}^{k l}=i\left(\boldsymbol{v}_{k l} \cdot \hat{\boldsymbol{r}}_{k l}\right) \Theta\left(-\boldsymbol{v}_{k l} \cdot \hat{\boldsymbol{r}}_{k l}\right) \delta\left(\left|\boldsymbol{r}_{k l}\right|-2 a\right)\left(b_{+}^{k l}-1\right) .
$$

The operator $b_{+}^{k l}$ replaces the linear and angular momenta of the two particles $k$ and $l$ before collision by the corresponding ones after collision, according to Eqs. (3) and (4). $\Theta(x)$ is the Heaviside step function, and we have introduced the notation $\boldsymbol{r}_{k l}=\boldsymbol{r}_{k}-\boldsymbol{r}_{l}$ and $\hat{\boldsymbol{r}}_{k l}=\boldsymbol{r}_{k l} /\left|\boldsymbol{r}_{k l}\right|$. Equation (A3) has the following interpretation: The factor $\boldsymbol{v}_{k l} \cdot \hat{\boldsymbol{r}}_{k l}$ gives the flux of incoming particles, while the $\Theta$ - and $\delta$-functions specify the conditions for a collision to take place. A collision between particles $k$ and $l$ happens only if the two particles are approaching each other which is ensured by $\Theta\left(-\boldsymbol{v}_{k l} \cdot \hat{\boldsymbol{r}}_{k l}\right)$. At the instant of a collision the distance between the two particles has to vanish when two particles touch, which is expressed by $\delta\left(\left|\boldsymbol{r}_{k l}\right|-2 a\right)$. Finally, $\left(b_{+}^{k l}-1\right)$ generates the change of linear and angular momenta according to Eqs. (3) and (4).

The ensemble average, $\langle\ldots\rangle_{t}$, of a dynamic variable, $A$, is defined by

$$
\begin{aligned}
\langle A\rangle_{t} & =\int d \Gamma \rho(0) A(t)=\int d \Gamma \rho(t) A(0) \\
& =\int \prod_{k=1}^{N}\left(d^{2} r_{k} d^{2} v_{k} d \omega_{k}\right) \rho(t) A(0) .
\end{aligned}
$$

Here $\rho(t)=\exp \left(-i \mathcal{L}_{+}^{\dagger} t\right) \rho(0)$ is the $N$-particle distribution function, whose time development is governed by the adjoint $\mathcal{L}_{+}^{\dagger}$ of the time evolution operator $\mathcal{L}_{+}$. Differentiating Eq. (A4) with respect to time yields

$$
\begin{aligned}
\frac{d}{d t}\langle A\rangle_{t} & =\int d \Gamma \rho(0) \frac{d}{d t} A(t)=\int d \Gamma \rho(0) i \mathcal{L}_{+} A(t) \\
& =\int d \Gamma \rho(0) \exp \left(i \mathcal{L}_{+} t\right) i \mathcal{L}_{+} A(0)=\int d \Gamma \rho(t) i \mathcal{L}_{+} A(0) \\
& =\left\langle i \mathcal{L}_{+} A\right\rangle_{t} .
\end{aligned}
$$

The observables of interest are the averaged energies per particle, or, more specifically, the granular temperatures for the two-dimensional system

$$
T_{\mathrm{tr}}:=\frac{E_{\mathrm{tr}}}{N}=\frac{1}{N} \sum_{k=1}^{N} \frac{m}{2}\left|\boldsymbol{v}_{k}\right|^{2},
$$




$$
\frac{1}{2} T_{\mathrm{rot}}:=E_{\mathrm{rot}}=\frac{1}{N} \sum_{k=1}^{N} \frac{I}{2}\left|\boldsymbol{\omega}_{k}\right|^{2}
$$

and the total kinetic energy $E=E_{\mathrm{tr}}+E_{\mathrm{rot}}$. To make the temperatures dimensionless we may choose to measure mass in units of the particle mass, and velocities in units of the driving velocity $v_{0}$ defined in Eq. (7).

Assuming a homogeneous density distribution and Gaussian velocity distributions the $N$-particle distribution function is given by

$$
\rho(t) \propto \prod_{k<l} \Theta\left(\left|\boldsymbol{r}_{k l}\right|-2 a\right) \exp \left\{-\left(\frac{E_{\mathrm{tr}}}{T_{\mathrm{tr}}(t)}+\frac{E_{\mathrm{rot}}}{T_{\mathrm{rot}}(t)}\right)\right\},
$$

where the product of Heaviside functions accounts for the excluded volume. Hence we get two coupled differential equations for the time evolution of the translational and rotational energies

$$
\begin{aligned}
& \frac{d}{d t} T_{\mathrm{tr}}(t)=\frac{d}{d t}\left\langle E_{\mathrm{tr}}\right\rangle_{t}=\left\langle i \mathcal{L}_{+} E_{\mathrm{tr}}\right\rangle_{t}=H_{\mathrm{dr}}+\left\langle i \mathcal{L}^{\prime}{ }_{+} E_{\mathrm{tr}}\right\rangle_{t}, \\
& \frac{1}{2} \frac{d}{d t} T_{\mathrm{rot}}(t)=\frac{d}{d t}\left\langle E_{\mathrm{rot}}\right\rangle_{t}=\left\langle i \mathcal{L}_{+} E_{\mathrm{rot}}\right\rangle_{t}=\left\langle i \mathcal{L}^{\prime}{ }_{+} E_{\mathrm{rot}}\right\rangle_{t} .
\end{aligned}
$$

The averages on the right-hand sides can be calculated as follows. These calculations are almost identical for the translational and rotational energies, so we will show in detail the time derivative of $T_{\mathrm{tr}}(t)$ only:

$$
\begin{aligned}
\left\langle i \mathcal{L}^{\prime}{ }_{+} E_{\mathrm{tr}}\right\rangle_{t}= & \left\langle\frac{1}{2} \sum_{k \neq l} i T_{+}^{k l} E_{\mathrm{tr}}\right\rangle_{t} \\
= & \frac{1}{2 N} \sum_{k=1}^{N} \sum_{\substack{l=1 \\
l \neq k}}^{N} \frac{m}{2} \prod_{j=1}^{N} d^{2} r_{j} d^{2} v_{j} d \omega_{j} \rho(\boldsymbol{r}, \boldsymbol{v}, \boldsymbol{\omega}) \\
& \left.\times i T_{+}^{k l}\left|\boldsymbol{v}_{k}\right|^{2}+\left|\boldsymbol{v}_{l}\right|^{2}\right)
\end{aligned}
$$

We have used that the binary collision operator $i T_{+}^{k l}$ yields zero acting on any variable other than the ones of the two particles involved in the collision. Defining

$$
\begin{aligned}
d \Gamma:= & \prod_{j=1}^{N} d^{2} r_{j} d^{2} v_{j} d \omega_{j} \prod_{l \neq j} \Theta\left(\left|\boldsymbol{r}_{j l}\right|-2 a\right) \\
& \times \exp \left(-\sum_{k=1}^{N} \frac{m}{2 T_{\text {tr }}(t)}\left|\boldsymbol{v}_{k}\right|^{2}-\sum_{k=1}^{N} \frac{I}{2 T_{\text {rot }}(t)}\left|\boldsymbol{\omega}_{k}\right|^{2}\right)
\end{aligned}
$$

and using the definition of $i T_{+}^{12}$ we can write

$$
\begin{aligned}
\left\langle i \mathcal{L}^{\prime}{ }_{+} E_{\mathrm{tr}}\right\rangle_{t}= & -\frac{N-1}{2 \int d \Gamma} \int d \Gamma\left(\hat{\boldsymbol{r}}_{12} \cdot \boldsymbol{v}_{12}\right) \delta\left(\left|\boldsymbol{r}_{12}\right|-2 a\right) \\
& \times \Theta\left(-\hat{\boldsymbol{r}}_{12} \cdot \hat{\boldsymbol{v}}_{12}\right)\left(b_{+}^{12}-1\right) \frac{m}{2}\left(\left|\boldsymbol{v}_{1}\right|^{2}+\left|\boldsymbol{v}_{2}\right|^{2}\right) .
\end{aligned}
$$

The change of energy $\Delta E_{\mathrm{tr}}:=(m / 2)\left(b_{+}^{12}-1\right)\left(\left|\boldsymbol{v}_{1}\right|^{2}+\left|\boldsymbol{v}_{2}\right|^{2}\right)$ that results from a collision of particle 1 and 2 depends only on the phase space variables of particle 1 and 2 . Since we assume spatial homogeneity this change of energy can only depend on the relative distance vector $\boldsymbol{r}_{12}:=\boldsymbol{r}_{1}-\boldsymbol{r}_{2}$ as well as the relative translational and rotational velocities $\boldsymbol{v}_{12}:=\boldsymbol{v}_{1}$ $-\boldsymbol{v}_{2}$ and $\boldsymbol{\omega}_{12}:=\boldsymbol{\omega}_{1}+\boldsymbol{\omega}_{2}$. Further, we assume instantaneous collisions. Therefore the change of energy can only depend on the direction of the distance vector $\hat{\boldsymbol{r}}_{12}=\left(\boldsymbol{r}_{1}-\boldsymbol{r}_{2}\right) /\left|\boldsymbol{r}_{1}-\boldsymbol{r}_{2}\right|$. Now we can perform the integrations over those particles that are not involved in the collision. The integrals over $d^{2} \boldsymbol{v}_{3}, \ldots, d^{2} \boldsymbol{v}_{N}$ and $d^{2} \boldsymbol{\omega}_{3}, \ldots, d^{2} \boldsymbol{\omega}_{N}$ are simple Gaussians. To integrate over $d^{2} \boldsymbol{r}_{3}, \ldots, d^{2} \boldsymbol{r}_{N}$ we introduce two more twodimensional integrals

$$
\int d^{2} \boldsymbol{R}_{1} d^{2} \boldsymbol{R}_{2} \delta^{2}\left(\boldsymbol{R}_{1}-\boldsymbol{r}_{1}\right) \delta^{2}\left(\boldsymbol{R}_{2}-\boldsymbol{r}_{2}\right),
$$

over two-dimensional $\delta$ functions, $\delta^{2}(\boldsymbol{r}):=\delta\left(r_{x}\right) \delta\left(r_{y}\right)$. Using the definition of the pair correlation function

$$
\frac{g\left(\left|\boldsymbol{R}_{1}-\boldsymbol{R}_{2}\right|\right)}{V^{2}}:=\frac{\int \prod_{j=1}^{N} d^{2} r_{j} \prod_{l \neq j} \Theta\left(\left|\boldsymbol{r}_{j l}\right|-2 a\right) \delta^{2}\left(\boldsymbol{R}_{1}-\boldsymbol{r}_{1}\right) \delta^{2}\left(\boldsymbol{R}_{2}-\boldsymbol{r}_{2}\right)}{\int \prod_{j=1}^{N} d^{2} r_{j} \prod \Theta\left(\left|\boldsymbol{r}_{j l}\right|-2 a\right)},
$$

where $V$ is the area of the system, we obtain

$$
\begin{aligned}
\left\langle i \mathcal{L}^{\prime}{ }_{+} E_{\mathrm{tr}}\right\rangle_{t}= & -\frac{N-1}{2 V^{2}}\left(\frac{m}{2 \pi T_{\mathrm{tr}}(t)}\right)^{2}\left(\frac{I}{2 \pi T_{\mathrm{rot}}(t)}\right) \int d^{2} R_{1} d^{2} R_{2} d^{2} v_{1} d^{2} v_{2} d^{2} \omega_{1} d \omega_{2} g\left(\left|\boldsymbol{R}_{12}\right|\right) \\
& \times \exp \left(\frac{-\frac{m}{2}\left(\left|\boldsymbol{v}_{1}\right|^{2}+\left|\boldsymbol{v}_{2}\right|^{2}\right)}{T_{\mathrm{tr}}(t)}-\frac{\frac{I}{2}\left(\left|\boldsymbol{\omega}_{1}\right|^{2}+\left|\boldsymbol{\omega}_{2}\right|^{2}\right)}{T_{\mathrm{rot}}(t)}\right) \times\left(\hat{\boldsymbol{R}}_{12} \cdot \boldsymbol{v}_{12}\right) \delta\left(\left|\boldsymbol{R}_{12}\right|-2 a\right) \Theta\left(-\hat{\boldsymbol{R}}_{12} \cdot \hat{\boldsymbol{v}}_{12}\right) \Delta E_{\mathrm{tr}} .
\end{aligned}
$$


Since the change of energy $\Delta E_{\text {tr }}$ depends only on $\boldsymbol{R}_{12}:=\boldsymbol{R}_{1}$ $-\boldsymbol{R}_{2}, \boldsymbol{v}_{12}$, and $\boldsymbol{\omega}_{12}$, we introduce the variables

$$
\begin{aligned}
& r:=R_{1}-R_{2}, \quad \boldsymbol{v}:=\frac{\boldsymbol{v}_{1}-\boldsymbol{v}_{2}}{\sqrt{2}}, \quad \boldsymbol{\omega}:=\frac{\boldsymbol{\omega}_{1}+\boldsymbol{\omega}_{2}}{\sqrt{2}}, \\
& \boldsymbol{R}:=\boldsymbol{R}_{1}, \quad \boldsymbol{V}:=\frac{\boldsymbol{v}_{1}+\boldsymbol{v}_{2}}{\sqrt{2}}, \quad \boldsymbol{\Omega}:=\frac{\boldsymbol{\omega}_{1}-\boldsymbol{\omega}_{2}}{\sqrt{2}} .
\end{aligned}
$$

The Jacobian of this transformation is 1 . The expression to integrate over is independent of $\boldsymbol{R}$ such that integration over $d^{2} R$ yields the area $V$. We write $\boldsymbol{r}$ in polar coordinates $(r, \phi)$ and can integrate over $d r$. Then, we choose the coordinate system for integrations over $d^{2} v$ such that the unit vector $\hat{\boldsymbol{r}}$ points in the $y$ direction. That means we can replace $\hat{\boldsymbol{r}}$ by the unit vector in the $y$ direction $\hat{\boldsymbol{e}}_{y}$ and integrate over $d \phi$ which simply yields $2 \pi$. For readability, we use now the unit vector $\hat{\boldsymbol{n}}$ instead of $\hat{\boldsymbol{e}}_{y}$. The integrals over $d^{2} V$ and $d \Omega$ are Gaussians, so that we obtain

$$
\begin{aligned}
\left\langle i \mathcal{L}^{\prime}{ }_{+} E_{\mathrm{tr}}\right\rangle_{t}= & -2 \pi \sqrt{2} a n_{0} g(2 a)\left(\frac{m}{2 \pi T_{\mathrm{tr}}(t)}\right)\left(\frac{I}{2 \pi T_{\mathrm{rot}}(t)}\right)^{1 / 2} \\
& \times \int d v_{1} d v_{2} d \omega \exp \left(\frac{-\frac{m}{2}\left[v_{1}^{2}+v_{2}^{2}\right]}{T_{\mathrm{tr}}(t)}+\frac{-\frac{I}{2}|\omega|^{2}}{T_{\mathrm{rot}}(t)}\right) \\
& \times(\hat{\boldsymbol{n}} \cdot \boldsymbol{v}) \Theta(-\hat{\boldsymbol{n}} \cdot \hat{\boldsymbol{v}}) \Delta E_{\mathrm{tr}},
\end{aligned}
$$

with the number density $n_{0}:=(N-1) / V \approx N / V$.

To solve the integrals above we need to take a look at the change of energy

$$
\begin{aligned}
\frac{2}{m} \Delta E_{\mathrm{tr}}:= & \left(b_{+}^{12}-1\right)\left(\left|\boldsymbol{v}_{1}\right|^{2}+\left|\boldsymbol{v}_{2}\right|^{2}\right) \\
= & \left(\left|\boldsymbol{v}^{\prime}{ }_{1}\right|^{2}+\left|\boldsymbol{v}^{\prime}{ }_{2}\right|^{2}\right)-\left(\left|\boldsymbol{v}_{1}\right|^{2}+\left|\boldsymbol{v}_{2}\right|^{2}\right) \\
(3),(4) & 4 \eta(\eta-1)\left[|\boldsymbol{v}|^{2}-(\hat{\boldsymbol{n}} \cdot \boldsymbol{v})^{2}\right]-\left(1-r^{2}\right)(\hat{\boldsymbol{n}} \cdot \boldsymbol{v})^{2} \\
= & 4(2 a \eta)^{2}|\hat{\boldsymbol{n}} \times \boldsymbol{\omega}|^{2}+4 a \eta(2 \eta-1)(\hat{\boldsymbol{n}} \times \boldsymbol{\omega}) \cdot \boldsymbol{v} \\
& +(2)
\end{aligned}
$$

with $\eta$ and $r_{t}(\gamma)$ given by Eqs. (6) and (19). Keep in mind that $\underline{\boldsymbol{v}}$ and $\boldsymbol{\omega}$ have been defined as $\boldsymbol{v}:=\boldsymbol{v}_{12} / \sqrt{2}$ and $\boldsymbol{\omega}:=\boldsymbol{\omega}_{12} / \sqrt{2}$. The difference in calculation for models $\mathrm{D}$ and E comes into play at this step. [For model D, at this point of the calculation, we would express $\boldsymbol{v}$ in polar coordinates $\left(v, \gamma_{12}\right)$ and insert $\eta=\eta\left(\gamma_{12}\right)$ instead of $\eta=\eta(\gamma)$ "assuming" that $\gamma \approx \gamma_{12}$. That way all integrals become Gaussians and can easily be solved. In particular, the integrals over the last term in Eq. (A12) vanish.] However, we will now go on with model E. To perform the integrations over $\boldsymbol{v}$ and $\boldsymbol{\omega}$ we substitute

$$
\begin{aligned}
& \boldsymbol{\omega}_{\perp}:=\sqrt{2}(\hat{\boldsymbol{n}} \times \boldsymbol{\omega})=(\sqrt{2} \omega, 0,0), \\
& \boldsymbol{g}:=\sqrt{2}[\boldsymbol{v}+a(\hat{\boldsymbol{n}} \times \boldsymbol{\omega})],
\end{aligned}
$$

thus introducing the relative velocity of the contact point $g$ as defined in Eq. (2). The vector $\boldsymbol{\omega}_{\perp}$ points in the $x$ direction (due to our choice of coordinates) and $\left|\boldsymbol{\omega}_{\perp}\right|=\left|\boldsymbol{\omega}_{12}\right|$, so that $\boldsymbol{v}_{\text {rot }}=a \boldsymbol{\omega}_{\perp}$. The Jacobian of this transformation is $2^{-3 / 2}$. In terms of these new variables $\Delta E_{\mathrm{tr}}$ reads

$$
\begin{aligned}
\frac{2}{m} \Delta E_{\mathrm{tr}}= & 2 \eta(\eta-1)\left[|\boldsymbol{g}|^{2}-(\hat{\boldsymbol{n}} \cdot \boldsymbol{g})^{2}\right]-\frac{1-r^{2}}{2}(\hat{\boldsymbol{n}} \cdot \boldsymbol{g})^{2} \\
& +2 a \eta \boldsymbol{g} \cdot \boldsymbol{\omega}_{\perp},
\end{aligned}
$$

and we get

$$
\begin{aligned}
\left\langle i \mathcal{L}_{+}^{\prime} E_{\mathrm{tr}}\right\rangle_{t}= & -\frac{2}{\sqrt{\pi}} a n_{0} g(2 a)\left(\frac{m}{4 T_{\mathrm{tr}}(t)}\right)\left(\frac{I}{4 T_{\mathrm{rot}}(t)}\right)^{1 / 2} \\
& \times \int d^{2} g d \omega_{\perp}(\hat{\boldsymbol{n}} \cdot \boldsymbol{g}) \Theta(-\hat{\boldsymbol{n}} \cdot \hat{\boldsymbol{g}}) \\
& \times \exp \left(-\frac{q m a^{2}}{4 T_{\mathrm{rot}}(t)}\left|\boldsymbol{\omega}_{\perp}\right|^{2}\right) \\
& \times \exp \left(-\frac{m\left[|\boldsymbol{g}|^{2}-2 a \boldsymbol{g} \cdot \boldsymbol{\omega}_{\perp}+a^{2}\left|\boldsymbol{\omega}_{\perp}\right|^{2}\right]}{4 T_{\mathrm{tr}}(t)}\right) \Delta E_{\mathrm{tr}} .
\end{aligned}
$$

Next, we express $\boldsymbol{g}$ in polar coordinates $(g, \gamma)$ where $\gamma$ is not the usual angle between $\boldsymbol{g}$ and $\hat{\boldsymbol{e}}_{x}$ but instead-as needed for incorporating Coulomb friction-the angle between $\boldsymbol{g}$ and $\hat{\boldsymbol{n}}$ [i.e., the angle between $\boldsymbol{g}$ and $\hat{\boldsymbol{e}}_{y}$, i.e., $\left.\boldsymbol{g}=(-g \sin \gamma, g \cos \gamma)\right]$. Expression (A14) reads now

$$
\begin{aligned}
\frac{2}{m} \Delta E_{\mathrm{tr}}= & 2 \eta(\eta-1) g^{2} \sin ^{2} \gamma-\frac{1-r^{2}}{2} g^{2} \cos ^{2} \gamma \\
& -2 a \eta g \omega_{\perp} \sin \gamma
\end{aligned}
$$

[note that $\eta=\eta(\cot \gamma)$ in the Coulomb friction case] and

$$
\begin{aligned}
\left\langle i \mathcal{L}_{+}^{\prime} E_{\mathrm{tr}}\right\rangle_{t}= & -\frac{2}{\sqrt{\pi} a} n_{0} g(2 a)\left(\frac{m}{4 T_{\mathrm{tr}}(t)}\right)\left(\frac{I}{4 T_{\mathrm{rot}}(t)}\right)^{1 / 2} \\
& \times \int_{\pi / 2}^{(3 / 2) \pi} d \gamma \int_{0}^{\infty} d g \int_{-\infty}^{\infty} d \omega_{\perp} g^{2} \cos \gamma \\
& \times \exp \left(-\frac{q m a^{2} \omega_{\perp}^{2}}{4 T_{\mathrm{rot}}(t)}\right) \\
& \times \exp \left(-\frac{m}{4 T_{\mathrm{tr}}(t)}\left[g^{2}+2 a g \omega_{\perp} \sin \gamma+a^{2} \omega_{\perp}^{2}\right]\right) \Delta E_{\mathrm{tr}} .
\end{aligned}
$$

Now we define $\mathcal{A}:=\left[m a^{2} / 4\right]\left[1 / T_{\text {tr }}(t)+q / T_{\text {rot }}(t)\right], \quad \mathcal{B}$ $:=m a \sin \gamma /\left[4 \mathcal{A} T_{\text {tr }}(t)\right]$ and substitute $p:=\sqrt{\mathcal{A}\left(\omega_{\perp}+\mathcal{B} g\right) \text { for }}$ $\omega_{\perp}$. This leads to Gaussian integrals over $p$ and $g$. Using $x^{2}:=1+\left(T_{\text {rot }}(t) / q T_{\text {tr }}(t)\right)$, we obtain

$$
\begin{aligned}
\left\langle i \mathcal{L}_{+}^{\prime} E_{\mathrm{tr}}\right\rangle_{t}= & -\frac{3}{2} \sqrt{\frac{\pi}{m}} 2 a n_{0} g(2 a) T_{\mathrm{tr}}^{3 / 2}(t) x^{4} \\
& \times \int_{\pi / 2}^{\pi} d \gamma \frac{\cos \gamma}{\left[1+\left(x^{2}-1\right) \cos ^{2} \gamma\right]^{5 / 2}} \\
& \times\left(4 \eta\left[\eta-\frac{1}{x^{2}}\right] \sin ^{2} \gamma-\left(1-r^{2}\right) \cos ^{2} \gamma\right) .
\end{aligned}
$$

Up to this point we have not specified, whether we are going to use constant coefficients of restitution or Coulomb friction. All this is hidden in $\eta$ which is either a constant or a 
function of $\gamma$. Since we are interested in the Coulomb friction case, we use $\eta=\eta(\gamma)$. We introduce the notation $\eta=[(1+r) / 2] \mu \min \left\{\left|\cot \gamma_{0}\right|,|\cot \gamma|\right\}$

and obtain

$$
\equiv \min \left\{\eta_{0},[(1+r) / 2] \mu|\cot \gamma|\right\},
$$

$$
\begin{aligned}
\left\langle i \mathcal{L}_{+}^{\prime} E_{\mathrm{tr}}\right\rangle_{t}= & -\frac{3}{2} \sqrt{\frac{\pi}{m}} 2 a n_{0} g(2 a) T_{\mathrm{tr}}^{3 / 2}(t) x^{4}\left\{\frac{2}{3} \frac{1-r^{2}}{x^{4}}\right. \\
& +\int_{\pi / 2}^{\gamma_{0}} d \gamma \frac{\cos \gamma}{\left[1+\left(x^{2}-1\right) \cos ^{2} \gamma\right]^{5 / 2}} \\
& \times\left(2 \mu \frac{1+r}{x^{2}} \sin \gamma \cos \gamma+\mu^{2}(1+r)^{2} \cos ^{2} \gamma\right) \\
& +4 \eta_{0}\left(\eta_{0}-\frac{1}{x^{2}}\right) \\
& \left.\times \int_{\gamma_{0}}^{\pi} d \gamma \frac{\cos \gamma \sin ^{2} \gamma}{\left[1+\left(x^{2}-1\right) \cos ^{2} \gamma\right]^{5 / 2}}\right\} .
\end{aligned}
$$

After performing the last integration the result can be written in the form

$$
\begin{aligned}
\frac{d}{d t} T_{\mathrm{tr}}(t)= & H_{\mathrm{dr}}-G T_{\mathrm{tr}}^{3 / 2}\left\{A_{r}+\frac{\eta_{0}}{2} \frac{1-\eta_{0} x^{2}}{\left(1+x^{2} \cot ^{2} \gamma_{0}\right)^{3 / 2}}\right. \\
& +\frac{\eta_{0}}{2} \frac{x^{2} \cot ^{2} \gamma_{0}}{\left(1+x^{2} \cot ^{2} \gamma_{0}\right)^{3 / 2}} \\
& \left.-\eta_{0}^{2} \tan ^{2} \gamma_{0}\left(1-\frac{1+\frac{3}{2} x^{2} \cot ^{2} \gamma_{0}}{\left(1+x^{2} \cot ^{2} \gamma_{0}\right)^{3 / 2}}\right)\right\},
\end{aligned}
$$

where $G=8 \sqrt{\pi / m} a n_{0} g(2 a)$, which is the same as Eq. (14), and $A_{r}=\left(1-r^{2}\right) / 4$. Similarly, $T_{\text {rot }}$ can be calculated using, instead of $\Delta E_{\mathrm{tr}}$, the change of rotational energy at collision,

$$
\begin{aligned}
\frac{2}{I} \Delta E_{\mathrm{rot}}: & \left(b_{+}^{12}-1\right)\left(\left|\boldsymbol{\omega}_{1}\right|^{2}+\left|\boldsymbol{\omega}_{2}\right|^{2}\right) \\
= & \left(\left|\boldsymbol{\omega}^{\prime}{ }_{1}\right|^{2}+\left|\boldsymbol{\omega}_{2}^{\prime}{ }_{2}\right|^{2}\right)-\left(\left|\boldsymbol{\omega}_{1}\right|^{2}+\left|\boldsymbol{\omega}_{2}\right|^{2}\right) \\
(3),(4) & \frac{4 \eta^{2}}{a^{2} q^{2}}|\hat{\boldsymbol{n}} \times \boldsymbol{v}|^{2}+4 \frac{\eta}{q}\left(\frac{\eta}{q}-1\right)\left(|\boldsymbol{\omega}|^{2}\right. \\
& \left.-(\hat{\boldsymbol{n}} \cdot \boldsymbol{\omega})^{2}\right)-\frac{4 \eta}{a q}\left(2 \frac{\eta}{q}-1\right)(\hat{\boldsymbol{n}} \times \boldsymbol{v}) \cdot \boldsymbol{\omega},
\end{aligned}
$$

which can be reformulated as

$$
\frac{2}{m} \Delta E_{\mathrm{rot}}=\frac{2 \eta^{2}}{q}\left[|\boldsymbol{g}|^{2}-(\hat{\boldsymbol{n}} \cdot \boldsymbol{g})^{2}\right]-2 a \eta \boldsymbol{g} \cdot \boldsymbol{\omega}_{\perp},
$$

using the notation introduced in Eqs. (A11) and (A13). The calculation for the rotational temperature is identical to the one for the translational temperature just shown until Eq. (A15) into which we insert $\Delta E_{\text {rot }}$ from Eq. (A21) instead of $\Delta E_{\mathrm{tr}}$. Performing the integrals yields

$$
\begin{aligned}
\frac{1}{2} \frac{d}{d t} T_{\mathrm{rot}}(t)= & G T_{\mathrm{tr}}^{3 / 2}\left\{\frac{1}{\left(1+x^{2} \cot ^{2} \gamma_{0}\right)^{3 / 2}}\right. \\
& \times\left(\frac{\eta_{0}^{2} x^{2}}{2 q}-\left(x^{2}-1\right) \frac{\eta_{0}}{2}\left(1+x^{2} \cot ^{2} \gamma_{0}\right)\right) \\
& \left.+\frac{\eta_{0}^{2} \tan ^{2} \gamma_{0}}{q}\left(1-\frac{1+\frac{3}{2} x^{2} \cot ^{2} \gamma_{0}}{\left(1+x^{2} \cot ^{2} \gamma_{0}\right)^{3 / 2}}\right)\right\} .
\end{aligned}
$$

Finally, from Eqs. (A19) and (A22) the conversant reader may reproduce the transformation to the more convenient coefficients in Eqs. (33).

For comparison, we quote the equivalent results in three dimensions: ${ }^{13}$

$$
\begin{aligned}
\frac{3}{2} \frac{d}{d t} T_{\mathrm{tr}}(t)= & H_{\mathrm{dr}}-G_{3 D} T_{\mathrm{tr}}^{3 / 2}\left\{A_{r}+\frac{\eta_{0}}{2} \frac{1-\eta_{0} x^{2}}{1+x^{2} \cot ^{2} \gamma_{0}}\right. \\
& \left.+\frac{\eta_{0}}{2}\left(\frac{\arctan \left(x \cot \gamma_{0}\right)}{x \cot \gamma_{0}}-\frac{1}{1+x^{2} \cot ^{2} \gamma_{0}}\right)\right\},
\end{aligned}
$$

and

$$
\begin{aligned}
\frac{3}{2} \frac{d}{d t} T_{\text {rot }}(t)= & G_{3 D} T_{\text {tr }}^{3 / 2}\left\{\eta_{0} \frac{1-\left(1-\frac{\eta_{0}}{q}\right) x^{2}}{1+x^{2} \cot ^{2} \gamma_{0}}-\frac{\eta_{0}}{2}\left(x^{2}-1\right)\right. \\
& \left.\times\left(\frac{\arctan \left(x \cot \gamma_{0}\right)}{x \cot \gamma_{0}}-\frac{1}{1+x^{2} \cot ^{2} \gamma_{0}}\right)\right\},(\mathrm{A}
\end{aligned}
$$

where $\quad G_{3 D}=32 \sqrt{\pi / m} a^{2} n_{0} g_{3 D}(2 a) \quad$ and $\quad g_{3 D}(2 a) \approx(1$ $-\nu / 2) /(1-\nu)^{3}$. $^{55}$

${ }^{1}$ Physics of Dry Granular Media —NATO ASI Series E350, edited by H. J. Herrmann, J.-P. Hovi, and S. Luding (Kluwer Academic, Dordrecht, 1998).

${ }^{2}$ Continuous and Discontinuous Modelling of Cohesive Frictional Materials, Lecture notes in Physics 568, edited by P. A. Vermeer, S. Diebels, W. Ehlers, H. J. Herrmann, S. Luding, and E. Ramm (Springer, Berlin, 2001).

${ }^{3}$ Powders \& Grains 2001, edited by Y. Kishino (Balkema, Rotterdam, 2001).

${ }^{4}$ Granular Gas Dynamics, edited by T. Pöschel and N. V. Brilliantov (Springer, Berlin, 2003).

${ }^{5}$ J. T. Jenkins and M. W. Richman, "Kinetic theory for plane shear flows of a dense gas of identical, rough, inelastic, circular disks," Phys. Fluids 28, 3485 (1985).

${ }^{6}$ C. K. K. Lun and S. B. Savage, "A simple kinetic theory for granular flow of rough, inelastic, spherical particles," J. Appl. Mech. 54, 47 (1987).

${ }^{7}$ C. K. K. Lun, "Kinetic theory for granular flow of dense, slightly inelastic, slightly rough spheres," J. Fluid Mech. 233, 539 (1991).

${ }^{8}$ A. Goldshtein and M. Shapiro, "Mechanics of collisional motion of granular materials. Part 1. General hydrodynamic equations," J. Fluid Mech. 282, 75 (1995).

${ }^{9}$ J. T. Jenkins and M. Y. Louge, "On the flux of fluctuation energy in a collisional grain flow at a flat, frictional wall," Phys. Fluids 9, 2835 (1997).

${ }^{10} \mathrm{M}$. Huthmann and A. Zippelius, "Dynamics of inelastically colliding rough spheres: Relaxation of translational and rotational energy," Phys. Rev. E 56, R6275 (1997).

${ }^{11}$ T. P. C. van Noije and M. H. Ernst, "Velocity distributions in homogeneous granular fluids: The free and the heated case," Granular Matter 1, 57 (1998).

${ }^{12}$ T. P. C. van Noije, M. H. Ernst, E. Trizac, and I. Pagonabarraga, "Randomly driven granular fluids: Large scale structure," Phys. Rev. E 59, 4326 (1999). 
${ }^{13}$ O. Herbst, M. Huthmann, and A. Zippelius, "Dynamics of inelastically colliding spheres with Coulomb friction: Relaxation of translational and rotational energy," Granular Matter 2, 211 (2000).

${ }^{14}$ J. T. Jenkins and C. Zhang, "Kinetic theory for identical, frictional, nearly elastic spheres," Phys. Fluids 14, 1228 (2002).

${ }^{15}$ S. Luding, "Driven granular gases," in Granular Gas Dynamics, edited by T. Pöschel and N. V. Brilliantov (Springer, Berlin, 2003), pp. 293-316.

${ }^{16}$ I. Goldhirsch, S. H. Noskowicz, and O. Bar-Lev "Weakly frictional granular gases," in Traffic and Granular Flow '03, edited by S. P. Hoogendoom, S. Luding, P. H. L. Bovy, M. Schreckenberg and D. E. Wolf (Springer, Berlin Heidelberg 2005), pp. 459-473; I. Goldhirsch, S. H. Noskowicz, and O. Bar-Lev, "Nearly smooth granular gases," Phys. Rev. Lett. 95, 068002 (2005).

${ }^{17}$ O. R. Walton and R. L. Braun, "Viscosity, granular-temperature, and stress calculations for shearing assemblies of inelastic, frictional disks," J. Rheol. 30, 949 (1986).

${ }^{18}$ C. S. Campbell, "Rapid granular flows," Annu. Rev. Fluid Mech. 22, 57 (1990).

${ }^{19}$ M. Y. Louge, "Computer simulations of rapid granular flows of spheres interacting with a flat, frictional boundary," Phys. Fluids 6, 2253 (1994).

${ }^{20}$ S. Luding, "Granular materials under vibration: Simulations of rotating spheres," Phys. Rev. E 52, 4442 (1995).

${ }^{21}$ S. Luding, M. Huthmann, S. McNamara, and A. Zippelius, "Homogeneous cooling of rough dissipative particles: Theory and simulations," Phys. Rev. E 58, 3416 (1998).

${ }^{22}$ S. McNamara and S. Luding, "Energy nonequipartition in systems of inelastic, rough spheres," Phys. Rev. E 58, 2247 (1998).

${ }^{23}$ S. J. Moon, J. B. Swift, and H. L. Swinney, "Role of friction in pattern formation in oscillated granular layers," Phys. Rev. E 69, 031301 (2004).

${ }^{24}$ J. S. Olafsen and J. S. Urbach, "Clustering, order and collapse in a driven granular monolayer," Phys. Rev. Lett. 81, 4369 (1998).

${ }^{25}$ J. S. Olafsen and J. S. Urbach, "Velocity distributions and density fluctuations in a 2d granular gas," Phys. Rev. E 60, R2468 (1999).

${ }^{26}$ W. Losert, D. G. W. Cooper, and J. P. Gollub, "Propagating front in an excited granular layer," Phys. Rev. E 59, 5855 (1999).

${ }^{27}$ G. W. Baxter and J. S. Olafsen, "Kinetics-Gaussian statistics in granular gases," Nature 425, 680 (2003).

${ }^{28}$ J.-C. Géminard and C. Laroche, "Pressure measurement in twodimensional horizontal granular gases," Phys. Rev. E 70, 021301 (2004).

${ }^{29}$ R. Cafiero, S. Luding, and H. J. Herrmann, "Two-dimensional granular gas of inelastic spheres with multiplicative driving," Phys. Rev. Lett. 84, 6014 (2000).

${ }^{30}$ S. F. Foerster, M. Y. Louge, H. Chang, and K. Allia, "Measurements of the collision properties of small spheres," Phys. Fluids 6, 108 (1994).

${ }^{31}$ S. Luding, E. Clément, A. Blumen, J. Rajchenbach, and J. Duran, "Interaction laws and the detachment effect in granular media," in Fractal Aspects of Materials,Symposium Proceedings (Materials Research Society, Pittsburgh, PA, 1995), Vol. 367, pp. 495-500.

${ }^{32}$ S. Luding, "Collisions \& contacts between two particles," in Physics of Dry Granular Media-NATO ASI Series E350, edited by H. J. Herrmann, J.-P. Hovi, and S. Luding (Kluwer Academic, Dordrecht, 1998), p. 285.

${ }^{33}$ B. D. Lubachevsky, "How to simulate billards and similar systems," J. Comput. Phys. 94, 255 (1991).
${ }^{34}$ O. R. Walton, "Numerical simulation of inelastic, frictional particleparticle interactions," in Particulate Two-phase Flow, edited by M. C. Roco (Butterworth-Heinemann, Boston, 1993), p. 884.

${ }^{35}$ L. Labous, A. D. Rosato, and R. Dave, "Measurements of collision properties of spheres using high-speed video analysis," Phys. Rev. E 56, 5715 (1997).

${ }^{36}$ S. Luding, O. Strauß, and S. McNamara, "Segregation of polydisperse granular media in the presence of a temperature gradient," in IUTAM Symposium on Segregation in Granular Flows, edited by T. Rosato (Kluwer Academic, Dordrecht, 1999), pp. 297-304.

${ }^{37}$ R. Soto, M. Mareschal, and D. Risso, "Departure from Fourier's law for fluidized granular media," Phys. Rev. Lett. 83, 5003 (1999).

${ }^{38}$ R. Soto and M. Mareschal, "Statistical mechanics of fluidized granular media: Short range velocity correlations," Phys. Rev. E 63, 041303 (2001).

${ }^{39}$ O. Herbst, P. Müller M. Otto, and A. Zippelius, "Local equation of state and velocity distributions of a driven granular gas," Phys. Rev. E 70, 051313 (2004).

${ }^{40}$ A. Puglisi, V. Loreto, U. M. B. Marconi, and A. Vulpiani, "Clustering and non-Gaussian behavior in granular matter," Phys. Rev. Lett. 81, 3848 (1998).

${ }^{41}$ C. Bizon, M. D. Shattuck, and J. B. Swift, "Linear stability analysis of a vertically oscillated granular layer," Phys. Rev. E 60, 7210 (1999).

${ }^{42}$ R. Cafiero, S. Luding, and H. J. Herrmann, "Rotationally driven gas of inelastic rough spheres," Europhys. Lett. 60, 854 (2002).

${ }^{43}$ B. D. Lubachevsky, F. H. Stillinger, and E. N. Pinson, "Disks vs. spheres: Contrasting properties of random packings," J. Stat. Phys. 64, 501 (1991).

${ }^{44}$ R. Mazighi, B. Bernu, and F. Delyon, "Steady state of a column of shaked inelastic spheres," Phys. Rev. E 50, 4551 (1994).

${ }^{45}$ S. McNamara and W. R. Young, "Inelastic collapse in two dimensions," Phys. Rev. E 50, R28 (1994).

${ }^{46}$ S. Luding, E. Clément, J. Rajchenbach, and J. Duran, "Simulations of pattern formation in vibrated granular media," Europhys. Lett. 36, 247 (1996).

${ }^{47}$ S. Luding and S. McNamara, "How to handle the inelastic collapse of a dissipative hard-sphere gas with the TC model," Granular Matter 1, 113 (1998).

${ }^{48}$ S. Luding and A. Goldshtein, "Collisional cooling with multi-particle interactions," Granular Matter 5, 159 (2003).

${ }^{49}$ D. Henderson, "A simple equation of state for hard discs," Mol. Phys. 30, 971 (1975).

${ }^{50} \mathrm{D}$. Henderson, "Monte Carlo and perturbation theory studies of the equation of state of the two-dimensional Lennard-Jones fluid," Mol. Phys. 34, 301 (1977).

${ }^{51}$ L. Verlet and D. Levesque, "Integral equations for classical fluids. III. The hard disks system," Mol. Phys. 46, 969 (1982).

${ }^{52} \mathrm{P}$. Sunthar and V. Kumaran, "Temperature scaling in a dense vibrofluidized granular material," Phys. Rev. E 60, 1951 (1999).

${ }^{53} \mathrm{D}$. K. Yoon and J. T. Jenkins, "Kinetic theory for identical, frictional, nearly elastic disks," Phys. Fluids 17, 083301 (2005).

${ }^{54}$ M. H. Ernst, J. R. Dorfman, W. R. Hoegy, and J. M. J. van Leeuwen, "Hard-sphere dynamics and binary-collision operators," Physica 45, 127 (1969).

${ }^{55}$ W. F. Carnahan and K. E. Starling, "Equation of state for nonattracting rigid spheres," J. Chem. Phys. 51, 635 (1969). 